COMMUNICATIONS IN

ANALYSIS AND GEOMETRY

Volume 11, Number 3, 391-423, 2003

\title{
Embedded Special Lagrangian Submanifolds in Calabi-Yau Manifolds
}

YNG-ING LEE

A Calabi-Yau manifold is a Kähler manifold with trivial canonical line bundle. It is proved by S.T. Yau [24] that in a Calabi-Yau manifold there exists a unique Ricci flat metric in its Kähler class. Therefore, we have two special forms $\omega$ and $\Omega$ in an n-dimensional Calabi-Yau manifold $N$, where $\omega$ is the Kähler form of the Ricci flat metric $g$ and $\Omega$ is a parallel holomorphic $(n, 0)$ form of unit length with respect to $g$. A real n-dimensional submanifold $L$ in $N$ is called Lagrangian if the restriction of $\omega$ on $L$ vanishes. If in addition, the restriction of $\operatorname{Im} \Omega$ on $L$ also vanishes, then $L$ is called special Lagrangian. This is equivalent to that $L$ is calibrated by Re $\Omega$. A calibrated submanifold is always volume minimizing. (See [7] or section 1 in this paper.) In particular, special Lagrangian submanifolds are minimal submanifolds of middle dimension. This motivates our study on special Lagrangian submanifolds or more generally on Lagrangian minimal submanifolds ([11], [12], [20]). Another motivation comes from mirror symmetry. In [23], A. Stominger, S.T. Yau, and E. Zaslow proposed to construct the mirror manifold of a Calabi-Yau manifold by the moduli space of special Lagrangian tori together with their flat connections. For development and modification of this conjecture, we refer to [9], [5], [17] etc., and the reference therein. The current paper is an attempt in employing the perturbation method to study problems in this direction. In particular, we prove

Theorem 3. Suppose that $L$ is a closed, connected, and immersed special Lagrangian submanifold in a closed Calabi-Yau manifold $N$ of complex dimension 3. Assume that $L$ has only isolated transversal self-intersection points. Then $L$ is the limit of a family of embedded closed special Lagrangian submanifolds in $N$.

Theorem 4. Suppose that $L$ is a closed, connected, and immersed special Lagrangian submanifold in a closed Calabi-Yau manifold $N$ of complex dimension $n>3$. Moreover, assume that $L$ has only isolated transversal self-intersection of two sheets and the two tangent planes at each intersection point satisfy the angle condition $\theta_{1}+\cdots+\theta_{n}=\frac{\pi}{2}$ (see section 2). Then 
$L$ is the limit of a family of embedded closed special Lagrangian submanifolds in $N$.

Remark: Theorem 4 does not hold if we drop the angle condition in the theorem. This is pointed out by N.C. Leung and is explained in section 2 .

I should mention the following result by A. Butscher:

Theorem (A. Butscher [3]). Suppose $L_{1}$ and $L_{2}$ are two special Lagrangian submanifolds with boundary of $R^{2 n}, n \geq 3$, that intersect transversally at one point $p$. Furthermore, suppose that the tangent planes of $L_{1}$ and $L_{2}$ satisfy the angle condition (as in above Theorem 4) and let $W$ be a scaffold for $L$ that is a codimension 2, symplectic submanifold of $R^{2 n}$. Define $L=L_{1} \cup L_{2}$. Then there exists a family $L_{\alpha}$ of smooth Lagrangian minimal submanifolds with boundary and a family of symplectic, codimension 2 submanifolds $W_{\alpha}$ such that the boundary of $L_{\alpha}$ lies in $W_{\alpha}$. Moreover, $L_{\alpha}$ and $W_{\alpha}$ converges to $L$ and $W$ respectively in some suitable topology.

The techniques in this paper and in A. Butscher's work are similar. We both choose a Lawlor neck (see [10] or section 1) as a local model, connect it to $L$ outside a small ball to construct approximate submanifolds which are Lagrangian, and then apply Hamiltonian deformation to perturb these approximate submanifolds to become special Lagrangian. The main difference between these two works is: In Butscher's situation, the set $L \backslash\{p\}$ has two connected components, and thus the first eigenvalues of the approximate submanifolds will tend to zero as the neck size tends to zero. To resolve this difficulty, Butscher allows the phase (see section 1) changing to have an extra freedom to do the perturbation. Hence $L_{\alpha}$ can only be Lagrangian minimal submanifolds instead of being special Lagrangian submanifolds (of the same phase) in his result. He also needs to allow the boundary moving to obtain $L_{\alpha}$. These are all necessary steps for the nature of his problem. In contrast to his situation, I have $L \backslash\{p\}$ connected and can prove that the first eigenvalues of the approximate submanifolds have an uniform positive lower bound (Theorem 1). In fact, a similar proof can also give a bound for the case that the singular set of $L$ is of codimension 2 in $L$. This observation is useful in generalizing the results to other cases. Because the first eigenvalues have an uniform positive lower bound, I do not need to change the phase to solve the problem and can obtain the deformation in the category of special Lagrangian submanifolds. This paper considers submanifolds in a general Calabi-Yau manifold instead of just in $R^{2 n}$. Therefore, it is also necessary to find nice coordinates balancing symplectic structure and complex structure, such that the local model can be adapted from $R^{2 n}$. This part becomes very 
delicate in cases that the intersection submanifolds are of higher dimension. Besides the above difference, I also prove that the angle condition is always satisfied when $n=2$ or 3 (see section 2 ). This is why we have a general theorem for dimensin 3. The 2-dimensional case needs a different treatment. We keep some discussions for this situation in a few places, but leave the complete proof to other paper.

It will be very interesting if one can construct new examples of special Lagrangian submanifolds by doing connected sum of two special Lagrangian submanifolds. Construction of approximate submanifolds in this situation is exactly the same. But as in Butscher's case, the first eigenvalues of the approximate submanifolds will tend to zero as the neck size tends to zero. By computing the dimension of local deformations of a special Lagrangian submanifold [13], it turns out that one cannot have an extra freedom to resolve a single intersection point of two special Lagrangian submanifolds. However, if they intersect at more than one point or consider a loop of special Lagrangian submanifolds which intersect consecutively, the topological obstruction will not happen. We need to analyze the perturbation in a different way because there is no positive lower bound for the first eigenvalues of the approximate submanifolds. This is an ongoing project with N.C. Leung.

Thank R. Schoen for bringing this interesting problem to my attention in the summer of 1998. I found that one could use Lawlor necks as local models immediately and began to study these problems. A. Butscher's thesis [2] was finished in the summer of 2000. The author thus referred most of the common part to his work. In preparing this paper, I found a few mistakes in A. Butscher's thesis, which include a wrong formula for the linearized operator (Proposition 4.28 in p.105), an incorrect argument for the sup. norm estimate (5.48 in p.142), and the necessity of adding the angle condition (Main Theorem 2 in p.5). A. Butscher gave a primary argument for the sup. norm estimate later [4]. Because it does not appear in other places, a treatment for cases considered in this paper is supplemented in the Appendix for the reader's reference.

I would like to thank the referee's suggestions on revising this paper and on making a comparison with A. Butscher's work. I also like to thank N.C. Leung, R. Schoen, C.L. Terng, and J. Wolfson for their discussions, as well as A. Butscher for sending me his papers and correcting my English in the first version of this paper. During the period of this research, I visited the National Center for Theoretical Sciences in Taiwan and Tom Wan in Chinese University, HongKong. I wish to express my gratitude to them for their 
hospitality and organizing stimulating mathematical activities. My research is partially supported by National Science Council in Taiwan with projects NSC 89-2115-M-002-018 and 90-2115-M-002-006.

The plan of this paper is as follows: In section 1, I explain Lawlor's examples (Lawlor necks) and give some basic definitions and properties. I prove in section 2 that the angle criterion is always satisfied when $n=2$ or 3 , and give an example to explain why Theorem 4 cannot hold in general. The construction of approximate submanifolds and an estimate on their first eigenvalues are given in section 3 . Finally in section 4 , I perturb the approximate submanifolds to become special Lagrangian and prove the main Theorems. Some additional estimates are supplemented in the Appendix. To make our presentation less messy, the constant $C$ in the paper may change in different contexts. Its dependency will be specified whenever it is essential.

\section{Preliminaries.}

Calibrated geometry and the notion of special Lagrangian submanifold were developed by R. Harvey and H. B. Lawson in [7]. We refer to their paper for a detailed discussion on this subject. The followings are some basic definitions:

Definition 1. A closed, differential p-form $\varphi$ on a Riemannian manifold $N$ is called a calibration if its comass is 1 . That is, $\varphi\left(e_{1}, \cdots, e_{p}\right) \leq 1$ for any oriented, orthonormal p-frame on $T N$ and the equality holds at some place.

Definition 2. We call a submanifold $M$ of $N$ being calibrated by $\varphi$ if $\varphi$ is a calibration and $\left.\varphi\right|_{M}=d V_{M}$, where $d V_{M}$ is the induced volume form on $M$.

A nice property of being calibrated is:

Proposition 1. [7] Suppose that $M$ is calibrated by $\varphi$, and is compact without boundary. Assume that $M^{\prime}$ is in the same homology class as $M$. Then one has $\operatorname{Vol}(M) \leq \mathrm{Vol}\left(M^{\prime}\right)$. The equality holds if and only if $M^{\prime}$ is also calibrated by $\varphi$.

Proof. Because $\varphi$ is a closed form, one has that $\int_{M} \varphi=\int_{M^{\prime}} \varphi$. Since $\left.\varphi\right|_{M}=d V_{M}$ and $\varphi\left(T M^{\prime}\right) \leq 1$, the inequality thus follows and equality holds if and only if $\varphi\left(T M^{\prime}\right)=1$, i.e., $M^{\prime}$ is also calibrated by $\varphi$. 
If $M$ has boundary and $\varphi$ is an exact form, one still can prove that $M$ has the least volume among all submanifolds with the same boundary. A pdimensional complex submanifold in a Kähler manifold $N$ is calibrated by $\frac{1}{p !} \omega^{p}$, where $\omega$ is the Kähler form on $N$, and hence is volume minimizing. R. Harvey and H. B. Lawson showed that the real part of $d Z=d z_{1} \wedge \cdots \wedge d z_{n}$, which is denoted by $R e d Z$, is a calibration in $R^{2 n}$. The corresponding calibrated submanifolds are called special Lagrangian. Let $\theta_{0}$ be a constant angle. The form $R e\left(e^{i \theta_{0}} d Z\right)$ is also a calibration in $R^{2 n}$, and its corresponding calibrated submanifolds are called special Lagrangian of phase $\theta_{0}$. These can be generalized to a Calabi-Yau manifold. Recall that there are two special forms $\omega$ and $\Omega$ in a complex n-dimensional Calabi-Yau manifold $N$, where $\omega$ is the Kähler form of the Ricci-flat metric and $\Omega$ is a parallel holomorphic $(n, 0)$ form of unit length. An n-dimensional submanifold $L$ in $N$ is called Lagrangian if the restriction of $\omega$ on $L$ vanishes. The $\mathrm{n}$-form $R e \Omega$ is a calibration and a Lagrangian submanifold in $N$ is called special Lagrangian if it is calibrated by $R e \Omega$. One can show that the restriction of $\Omega$ on a Lagrangian submanifold $L$ is equal to $e^{i \theta} d V_{L}$, where the value $e^{i \theta}$ depends on points. Hence being special Lagrangian is equivalent to that both the restriction of $\omega$ and $\operatorname{Im} \Omega$ (the imaginary part of $\Omega$ ) on $L$ vanish.

G. Lawlor [10] modified an example of R. Harvey and H. B. Lawson [7] and defined the following submanifolds, which will be called Lawlor necks in this paper:

Assume that $a_{1}, \cdots, a_{n}, n \geq 2$, are $\mathrm{n}$ positive real numbers and $a=$ $\left(a_{1}, \cdots, a_{n}\right)$. Set

$$
\theta_{k}(a, \mu)=\int_{0}^{\mu} \frac{a_{k} d s}{\left(1+a_{k} s^{2}\right) \sqrt{P(s)}} \text { for } \mu \geq 0,
$$

where

$$
P(s)=\frac{\left(1+a_{1} s^{2}\right) \cdots\left(1+a_{n} s^{2}\right)-1}{s^{2}} .
$$

Extend $\theta_{k}(a, \mu)$ to negative $\mu$ by $\theta_{k}(a,-\mu)=-\theta_{k}(a, \mu)$, and define a map $\Phi_{a}$ from $R \times S^{n-1}$ to $R^{2 n}$ by

$$
\Phi_{a}\left(\mu, x_{1}, \cdots, x_{n}\right)=\left(z_{1} x_{1}, \cdots, z_{n} x_{n}\right)
$$

where

$$
x_{1}^{2}+\cdots+x_{n}^{2}=1 \text { and } z_{k}=\sqrt{\frac{1}{a_{k}}+\mu^{2}} e^{i \theta_{k}(a, \mu)}
$$


Note that

$$
\Phi_{\frac{a}{t^{2}}}\left(\mu, x_{1}, \cdots, x_{n}\right)=t \Phi_{a}\left(\frac{\mu}{t}, x_{1}, \cdots, x_{n}\right) \text { for } t>0 .
$$

By scaling we can assume $\inf _{k=1, \cdots, n} a_{k}=1$. Denote

$$
\theta_{k}(a)=\int_{0}^{\infty} \frac{a_{k} d s}{\left(1+a_{k} s^{2}\right) \sqrt{P(s)}}, \quad \text { for } \quad k=1, \cdots, n
$$

It can be shown that $\theta_{1}(a)+\cdots+\theta_{n}(a)=\frac{\pi}{2}$. There is a bijection between positive $\theta_{1}, \cdots, \theta_{n}$ satisfying $\theta_{1}+\cdots+\theta_{n}=\frac{\pi}{2}$ and $a_{1}, \cdots, a_{n}$ satisfying $\inf _{k=1, \cdots, n} a_{k}=1$ such that $\theta_{i}=\theta_{i}(a)$ [10]. Denote the image of $\Phi_{a}$ by $M_{a}$. G. Lawlor proved that $M_{a}$ is embedded, calibrated by $\operatorname{Im} d Z$, and asymptotic to $P_{\theta}$ and $P_{-\theta}$, where $P_{\theta}$ is the plane

$$
P_{\theta}=\left\{\left(t_{1} e^{i \theta_{1}(a)}, \cdots, t_{n} e^{i \theta_{n}(a)}\right): t_{j} \in R, j=1, \cdots, n\right\} .
$$

Note that $M_{a}, P_{\theta}$ and $-P_{-\theta}$ are special Lagrangian of phase $\frac{\pi}{2}$, where $-P_{-\theta}$ is the plane $P_{-\theta}$ with orientation different from the standard one. By moving these spaces by a phase, we can always make them special Lagrangian. We thus will not specify the phase any more. But when we talk about special Lagrangian submanifolds in this paper, we do mean that they are calibrated by the same form, i.e. they are of the same phase. (see [2], [6], [8], [10]).

A. Butscher [2] studies carefully the asymptotic behavior of the above Lawlor neck. We summarize some of his results here for completeness. Note that $P(\mu) \leq \mu^{2 n-2}$ for $n \geq 2$. Thus one can prove that $\left|\theta_{k}(a, \mu)-\theta_{k}(a)\right| \leq \frac{1}{n|\mu|^{n}}$. Moreover, there exists a positive real number $R_{0}$ so that $M_{a} \backslash B_{R_{0}}(0)$ can be written as the graph of the gradient of a function

$$
\Psi: P_{i} \backslash B_{R_{0}}(0) \rightarrow R, \quad i=1,2 .
$$

Here we denote the two asymptotic planes of $M_{a}$ by $P_{1}$ and $P_{2}$, and split $R^{2 n}$ into $P_{i} \times P_{i}^{\perp}$ to write the graph. The function $\Psi$ has the properties that

$$
|\nabla \Psi(x)| \leq \frac{C}{|x|^{n-1}}, \quad\left|\nabla^{2} \Psi(x)\right| \leq \frac{C}{|x|^{n}}, \quad\left|\nabla^{3} \Psi(x)\right| \leq \frac{C}{|x|^{n+1}}
$$

and $\left|\nabla^{4} \Psi(x)\right| \leq \frac{C}{|x|^{n+2}}$ for $x \in P_{i}$ with $|x| \geq R_{0}$. The constant $C$ depends only on $a$ and $n$. The scaled manifold

$$
\varepsilon\left(M_{a} \backslash B_{R_{0}}(0)\right)=\varepsilon M_{a} \backslash B_{\varepsilon R_{0}}(0), \quad \varepsilon>0,
$$


is the graph of the gradient of a function

$$
\Psi_{\varepsilon}: P_{i} \backslash B_{\varepsilon R_{0}}(0) \rightarrow R, \quad i=1,2 .
$$

The function $\Psi_{\varepsilon}(x)=\varepsilon^{2} \Psi\left(\frac{x}{\varepsilon}\right)$ satisfies

$$
\left|\nabla \Psi_{\varepsilon}(x)\right| \leq \frac{C \varepsilon^{n}}{|x|^{n-1}}, \quad\left|\nabla^{2} \Psi_{\varepsilon}(x)\right| \leq \frac{C \varepsilon^{n}}{|x|^{n}}, \quad\left|\nabla^{3} \Psi_{\varepsilon}(x)\right| \leq \frac{C \varepsilon^{n}}{|x|^{n+1}},
$$

and $\left|\nabla^{4} \Psi_{\varepsilon}(x)\right| \leq \frac{C \varepsilon^{n}}{|x|^{n+2}}$ for $x \in P_{i}$ with $|x| \geq \varepsilon R_{0}$. We can assume further that $\left|\Psi_{\varepsilon}(x)\right| \leq \frac{C \varepsilon^{n}}{|x|^{n-2}}$ when $n \geq 3$ and $\left|\Psi_{\varepsilon}(x)\right| \leq C \varepsilon^{2} \ln \frac{|x|}{\varepsilon R_{0}}$ when $n=2$.

\section{Local model.}

Assume that $p$ is a transversal intersection point of two locally sheets of special Lagrangian submanifolds. We want to choose a Lawlor neck as a local model to resolve the intersection point and construct approximate submanifolds. The process is as follows: First find a Lawlor neck which is asymptotic to the two tangent planes at $p$. Then replace the two sheets of special Lagrangian submanifolds inside a small ball of $p$ by a scaled Lawlor neck, and connect the Lawlor neck to the original two sheets outside the ball. There is a condition $\theta_{1}+\cdots+\theta_{n}=\frac{\pi}{2}$ for the planes which Lawlor necks can be asymptotic to. In this section, we will show that this condition is always satisfied for our situation in dimension 2 and 3, but is not satisfied in general when $n \geq 4$. Hence when $n \geq 4$, we need to add the angle condition $\theta_{1}+\cdots+\theta_{n}=\frac{\pi}{2}$ in Theorem 4 . We also discuss why Theorem 4 cannot hold in general if $n \geq 4$.

Given a pair of Lagrangian planes $P_{1}$ and $P_{2}$ in $R^{2 n}$ passing the origin, we claim that one can find suitable coordinates, such that $P_{1}$ is the $x_{1}, \cdots, x_{n}$ plane and $P_{2}$ is the plane $\left\{\left(t_{1} e^{i \omega_{1}}, \cdots, t_{n} e^{i \omega_{n}}\right): t_{j} \in R, j=1, \cdots, n\right\}$, where

$$
0 \leq\left|\omega_{1}\right| \leq\left|\omega_{2}\right| \leq \cdots \leq\left|\omega_{n-1}\right| \leq \frac{\pi}{2} \quad \text { and } \quad\left|\omega_{n-1}\right| \leq\left|\omega_{n}\right| \leq \pi-\left|\omega_{n-1}\right| .
$$

A Lagrangian plane in $R^{2 n}$ passing the origin is the image of the real $x_{1}, \cdots, x_{n}$ plane under a linear transformation $A \in U(n)$. Thus the set of Lagrangian planes can be identified with $U(n) / S O(n)$ [7]. The Lie algebra $u(n)$ of $U(n)$ can be decomposed into the direct sum of $S$ and $s o(n)$, where $S$ is the set of pure imaginary symmetric matrices and $s o(n)$ is the set of real skew symmetric matrices. The subalgebra $S$ and $s o(n)$ corresponds 
to the -1 eigenspace and 1 eigenspace of the involution $\tau: u(n) \rightarrow u(n)$ respectively, where $\tau(y)=-y^{t}$. Since one can diagonalize a real symmetric matrix, it follows that $S=\cup k T k^{-1}$, where $T$ is a pure imaginary diagonal matrix and $k$ is in $S O(n)$. The symmetric space $U(n) / S O(n)$ is exactly exp $S$. Permute the coordinates to satisfy the condition on $\omega_{j}$. We thus prove the claim as desired. We like to thank C.L. Terng's help on this observation.

Denote $\left|\omega_{j}\right|$ by $\beta_{j}$. These angles satisfy

$$
0 \leq \beta_{1} \leq \beta_{2} \leq \cdots \leq \beta_{n-1} \leq \frac{\pi}{2} \quad \text { and } \quad \beta_{n-1} \leq \beta_{n} \leq \pi-\beta_{n-1} .
$$

They are exactly the characterizing angles between $P_{1}$ and $P_{2}$ as defined in [10]. Note that one has $0 \leq \sum_{j=1}^{n} \beta_{j}=\sum_{j=1}^{n}\left|\omega_{j}\right| \leq \frac{n \pi}{2}$, and $\beta_{j}>0$ if the pair of planes intersect only at the origin. Suppose that $P_{1}$ and $P_{2}$ are two special Lagrangian planes which intersect only at the origin. The special Lagrangian condition implies that $\omega_{j}$ satisfies $\sum_{j=1}^{n} \omega_{j}=2 k \pi$ for some integer $k$. Remember that $\sum_{j=1}^{n}\left|\omega_{j}\right| \leq \frac{n \pi}{2}$. It thus follows that $\sum_{j=1}^{n} \omega_{j}=0$ when $n=2$ or 3. It implies that $\beta_{1}=\beta_{2}$ in the case $n=2$, and $\beta_{1}+\beta_{2}=\beta_{3}$ in the case $n=3$. If we change the orientation on $P_{2}$, which is denoted by $-P_{2}$, then the characterizing angles between $P_{1}$ and $-P_{2}$ satisfy $\sum_{j=1}^{n} \bar{\beta}_{j}=\pi$ in the case $n=2$ or 3 . Change the coordinates such that $P_{1}=P_{\theta}$ and $-P_{2}=P_{-\theta}$, where $\theta=\left(\frac{\bar{\beta}_{1}}{2}, \frac{\bar{\beta}_{2}}{2}\right)$ or $\left(\frac{\bar{\beta}_{1}}{2}, \frac{\bar{\beta}_{2}}{2}, \frac{\bar{\beta}_{3}}{2}\right)$. We thus can find a Lawlor neck which is asymptotic to $P_{1}$ and $-P_{2}$. The angle condition is not always satisfied when $n \geq 4$. For example, the $x_{1}, \cdots, x_{4}$ plane and $y_{1}, \cdots, y_{4}$ plane in $R^{8}$ are two special Lagrangian planes which intersect only at the origin. But all the characterizing angles between them are $\frac{\pi}{2}$. Hence the sum of the angles is $2 \pi$ and there does not exist a Lawlor neck which is asymptotic to the $x_{1}, \cdots, x_{4}$ plane and $y_{1}, \cdots, y_{4}$ plane.

The geometric obstruction for finding a Lawlor neck in $n \geq 4$ comes from the following: There is an angle criterion which says that the nonzero sum (oriented union) $P_{1}+P_{2}$ is area minimizing if and only if the characterizing angles between them satisfy the inequality $\beta_{n} \leq \beta_{1}+\cdots+\beta_{n-1}$. (See [6], [10], [16].) Suppose that $P_{1}$ and $P_{2}$ are two special Lagrangian planes. By the property of calibration, we know that $P_{1}+P_{2}$ is area minimizing and thus $\beta_{n} \leq \beta_{1}+\cdots+\beta_{n-1}$. If $\beta_{n}<\beta_{1}+\cdots+\beta_{n-1}$, we can find two Lagrangian planes $P_{1}^{\prime}$ and $P_{2}^{\prime}$ near $P_{1}$ and $P_{2}$, which are not special Lagrangian and whose characterizing angles $\left\{\beta_{j}^{\prime}\right\}, j=1, \cdots, n$, still satisfy $\beta_{n}^{\prime}<\beta_{1}^{\prime}+\cdots+\beta_{n-1}^{\prime}$. Assume that there exists a special Lagrangian submanifold $L$ asymptotic to 
$P_{1}$ and $P_{2}$. Furthermore, assume that $L$ is the union of compact hypersurfaces in a family of Lagrangian planes. The last condition is equivalent to being a Lawlor neck. The intersection of $L$ and $P_{1}^{\prime}+P_{2}^{\prime}$ is a compact hypersurface in $P_{1}^{\prime}+P_{2}^{\prime}$. It is the boundary of a compact subset $A$ in $L$, and is also the boundary of a compact subset $E_{1}+E_{2}$ in $P_{1}^{\prime}+P_{2}^{\prime}$. By special Lagrangian condition on $L$ and applying angle criterion to $P_{1}^{\prime}+P_{2}^{\prime}$, we know that $A$ and $E_{1}+E_{2}$ are both volume minimizing and have the same boundary. It follows by Proposition 1 that they are calibrated by the same form, which is a contradiction because $P_{1}^{\prime}$ and $P_{2}^{\prime}$ are chosen to be not special Lagrangian. Thus we cannot have a Lawlor neck to approximate such a pair. Is it possible to find local models of different nature to resolve the isolated self-intersection point in this case? The answer is still no and is explained in next paragraph.

There is a $S^{2}$ family of compatible complex structures in a hyperkähler manifold. A complex Lagrangian submanifold in a hyperkähler manifold is a complex submanifold with respect to one of the compatible complex structures, and is special Lagrangian with respect to another compatible complex structure. By Proposition 1, a volume minimizing submanifold in the homology class of a calibrated submanifold must be calibrated by the same form. Thus all special Lagrangian submanifolds in the homology class of a complex Lagrangian submanifold are complex Lagrangian. If we can resolve the transversal intersection points of two special Lagrangian submanifolds in general, it implies that we can do the connected sum in the complex category in this case. However, it is known to be impossible when the complex dimension is bigger than one. Examples of closed, connected and immersed complex Lagrangian submanifolds with isolated transversal self-intersection points and of complex dimension bigger than one can be constructed. When we add a handle $\left(\cong S^{n-1} \times R, n \geq 4\right)$ to the complex Lagrangian submanifold, it will increase the dimension of the first homology group by one. This new topology cannot carry a Kähler structure since its first homology group is of odd dimension. Hence the theorem without further condition cannot hold when $n \geq 4$. It is still open whether the result holds for all odd $n$.

\section{Approximate submanifolds.}

Let $N$ be a closed Calabi-Yau manifold of dimension $n \geq 2$. Suppose that $L$ is an immersed special Lagrangian submanifold in $N$, which is closed, connected, and with only isolated transversal self-intersection points. Without loss of generality, we can assume that there is only one self-intersection point $p$ and locally $p$ is the transversal intersection of two sheets of $L$. In a small 
neighborhood of $p$, the metric in $N$ is equivalent to the Euclidean metric in $R^{2 n}$. For simplicity, the distance and norm in constructing approximate submanifolds are with respect to the Euclidean metric unless specified explicitly. We can choose coordinates $x_{1}, \cdots, x_{n}, y_{1}, \cdots, y_{n}$ and a ball $B_{r_{0}}(p)$ such that $p$ is the origin and for $q \in B_{r_{0}}(p)$ :

1. the Kähler form satisfies $\omega(q)=\sum_{i=1}^{i=n} d x_{i} \wedge d y_{i}$,

2. the metric $g=d s^{2}$ in $N$ satisfies $\left|d s^{2}(q)-d s_{0}^{2}\right| \leq C|q|^{2}$, where the metric $d s_{0}^{2}=\sum_{i=1}^{i=n} d x_{i}^{2}+d y_{i}^{2}$,

3. the complex structure $J$ satisfies $\left|J(q)-J_{0}\right| \leq C|q|^{2}$, where $J_{0}$ is the standard complex structure in $R^{2 n}$,

4. the parallel holomorphic $(n, 0)$ form $\Omega$ satisfies $|\Omega(q)-d Z| \leq C|q|^{2}$, where $d Z=d z_{1} \wedge \cdots \wedge d z_{n}$ and $z_{j}=x_{j}+i y_{j}, j=1, \cdots, n$.

To find coordinates satisfying these conditions, we can first choose normal coordinates at $p$ which satisfy condition 2, 3, and 4. Then apply Moser's techniques [15] to change the coordinates to become Darboux coordinates (condition 1). This process does not affect the property that the coordinates satisfy condition 2,3 , and 4 . Thus we obtain coordinates that satisfy the required conditions.

Denote the two tangent planes at $p$ by $P_{1}$ and $P_{2}$, which are special Lagrangian with respect to the standard structures in $R^{2 n}$. Because $L \cap B_{r_{0}}(p)$ is Lagrangian with respect to $\sum_{i=1}^{i=n} d x_{i} \wedge d y_{i}$, there exists $r_{1}<r_{0}$ such that $L \cap B_{r_{1}}(p)$ can be written as the graph of the gradient of a function

$$
\psi: P_{i} \cap B_{r_{1}}(p) \rightarrow R, \quad i=1,2 .
$$

Moreover, we can choose $\psi$ satisfying

$$
|\psi(x)| \leq K|x|^{3}, \quad|\nabla \psi(x)| \leq K|x|^{2}, \quad\left|\nabla^{2} \psi(x)\right| \leq K|x|, \quad\left|\nabla^{3} \psi(x)\right| \leq K,
$$

and $\left|\nabla^{4} \psi(x)\right| \leq K$ for $x \in P_{i}$ with $|x| \leq r_{1}$, where $K$ is a constant depending on the curvature of $L$ in $B_{r_{1}}(p)$.

When $P_{1}=P_{\theta},-P_{2}=P_{-\theta}$, and $\theta_{1}+\cdots+\theta_{n}=\frac{\pi}{2}$, there exists a Lawlor neck $M_{a}$ in $R^{2 n}$ asymptotic to $P_{1}$ and $P_{2}$. From discussions in section 2 , this 
condition is always satisfied when $n=2$ or 3 . From now on, we focus on the situations where we can find a Lawlor neck $M_{a}$ asymptotic to $P_{1}$ and $P_{2}$. Outside a small ball $B_{\varepsilon R_{0}}$, the scaled manifold $\varepsilon M_{a}$ can be written as the graph of the gradient of $\Psi_{\varepsilon}$ over $P_{1}$ and $P_{2}$. To match $\psi$ and $\Psi_{\varepsilon}$ together, one needs the following estimate:

Lemma 1. [2] When $n \geq 3$, there exist constants $\alpha_{0}$ and $c$ depending on $L$ only, such that if $0<\alpha<\alpha_{0}, r=\frac{\alpha}{K}$, and $\varepsilon<c \alpha^{1+\frac{1}{n}}$, then

$$
\left|\nabla^{2} \psi(x)\right| \leq \alpha \text { and }\left|\nabla^{2} \Psi_{\varepsilon}(x)\right| \leq \alpha \quad \text { for any } x \in P_{i} \quad \text { with } \frac{r}{2} \leq|x| \leq r .
$$

We will choose the approximate submanifolds to be $\varepsilon M_{a}$ near $p$, and to be $L$ outside a neighborhood of $p$. The interpolation between $\varepsilon M_{a}$ and $L$ is required to be Lagrangian. Recall that the graph of the gradient of a function on a Lagrangian plane is always Lagrangian. Hence the following combination of $\psi$ and $\Psi_{\varepsilon}$ is a good candidate for our purpose. Choose a smooth function $\eta$ on $R^{n}$ satisfying $0 \leq \eta(x) \leq 1, \eta(x) \equiv 1$ when $|x| \leq \frac{r}{2}$ and $\eta(x) \equiv 0$ when $|x| \geq \frac{3 r}{4}$. Moreover, we require $\eta(x)$ to satisfy

$$
|\nabla \eta(x)| \leq \frac{C}{r}, \quad\left|\nabla^{2} \eta(x)\right| \leq \frac{C}{r^{2}}, \quad\left|\nabla^{3} \eta(x)\right| \leq \frac{C}{r^{3}}, \quad \text { and } \quad\left|\nabla^{4} \eta(x)\right| \leq \frac{C}{r^{4}} .
$$

Define the interpolation to be the graph

$$
T_{i}=\left\{\left(x, \nabla\left[(1-\eta) \psi+\eta \Psi_{\varepsilon}\right](x)\right) \in P_{i} \times P_{i}^{\perp}, \frac{r}{2} \leq|x| \leq r\right\}, \quad i=1,2 .
$$

It is easy to check that

$$
\left|\nabla\left[(1-\eta) \psi+\eta \Psi_{\varepsilon}\right]\right|<C r^{2}, \text { for } \frac{r}{2} \leq|x| \leq r .
$$

Denote

$$
B_{r}^{\prime}=B_{r}^{P_{1}} \times R^{n} \cap B_{r}^{P_{2}} \times R^{n} \subset P_{1} \times P_{1}^{\perp} \cap P_{2} \times P_{2}^{\perp},
$$

where $B_{r}^{P_{i}}=B_{r} \cap P_{i}, i=1,2$. We then define the approximate submanifold to be

$$
M_{\alpha}=\left(\varepsilon M_{a} \cap B_{\frac{r}{2}}^{\prime}\right) \cup T_{1} \cup T_{2} \cup\left(L \backslash B_{r}^{\prime}\right) .
$$

The approximate submanifold is Lagrangian and satisfies the following properties:

$$
\begin{cases}|H(q)| \leq C|q| & \text { for } \quad q \in \varepsilon M_{a} \cap B_{\frac{r}{2}}^{\prime} \\ |H(q)| \leq C & \text { for } \quad q \in T_{1} \cup T_{2} \\ |H(q)|=0 & \text { for } \quad q \in L \backslash B_{r}^{\prime}\end{cases}
$$


where $H$ is the mean curvature vector of $M_{\alpha}$ in $N$. One also has

$$
\left\{\begin{array}{ll}
\left.|\operatorname{Im} \Omega|_{M_{\alpha}}(q)|\leq C| q\right|^{2} & \text { for } \quad q \in \varepsilon M_{a} \cap B_{\frac{r}{2}}^{\prime} \\
|\operatorname{Im} \Omega|_{M_{\alpha}}(q) \mid \leq C \alpha & \text { for } q \in T_{1} \cup T_{2} \\
|\operatorname{Im} \Omega|_{M_{\alpha}}(q) \mid=0 & \text { for } q \in L \backslash B_{r}^{\prime}
\end{array} .\right.
$$

The situation in $R^{2 n}$ is computed in [2]. Because $|\Omega(q)-d Z| \leq C|q|^{2}$ and $\left|H(q)-H_{0}(q)\right| \leq C|q|$, where $H_{0}$ is the mean curvature vector of $M_{\alpha} \cap B_{r}^{\prime}$ in $R^{2 n}$ with the Euclidean metric, we thus obtain the above estimates.

The approximate submanifolds $M_{\alpha}$ are embedded Lagrangian submanifolds. Because the mean curvature is uniformly bounded, by monotonicity formula [21] and the construction of $M_{\alpha}$, it follows that the area is uniformly bounded from above and below. For small $\alpha$, the homology classes of $M_{\alpha}$ and $L$ are equal and consequently $\int_{M_{\alpha}} \operatorname{Im} \Omega=0$. J. H. Michael and L. Simon [14] proved the Sobolev inequality:

$$
\left(\int_{M} h^{\frac{n}{n-1}} d V\right)^{\frac{n-1}{n}} \leq C(n) \int_{M}\left(\left|\nabla^{M} h\right|+h|\bar{H}|\right) d V
$$

for a submanifold $M^{n} \subset R^{l}$, where $h$ is a $C^{1}$ nonnegative function on $M$ with compact support and $\bar{H}$ is the mean curvature of $M$ in $R^{l}$. By embedding $N$ isometrically in $R^{l}$, the corresponding mean curvature $\bar{H}_{\alpha}$ of $M_{\alpha}$ in $R^{l}$ is uniformly bounded. Thus the norm of the Sobolev constant on $M_{\alpha}$ is uniformly bounded and one has the following lemma.

Lemma 5'. Suppose that $u$ is a positive sub-solution of the equation $\Delta_{M} u \geq g u$ on a closed manifold $M$, where $g$ is a $L^{1}$ function satisfying the estimate $\|g\|_{L^{\frac{r}{2}}} \leq \bar{c} \operatorname{Vol}(M)^{\frac{2}{r}-\frac{2}{n}}$ for some $r>n$. Then $\|u\|_{0, M} \leq C_{p} \operatorname{Vol}(M)^{-\frac{1}{p}}\|u\|_{L^{p}}$ for $p>0$. The constant $C_{p}$ depends on the Sobolov constant, $r, \bar{c}$ and $p$.

This lemma is a modification of Lemma 5 together with a remark after its proof, which is discussed in the Appendix. With Lemma 5', we can obtain an uniform positive lower bound for the first eigenvalue of $M_{\alpha}$.

Theorem 1. Suppose that $n$ is greater than 2. When $\alpha$ is small enough, the first eigenvalue $\lambda_{1}\left(M_{\alpha}\right)$ for the Laplace operator on $M_{\alpha}$ is bounded below by $\frac{1}{4} \lambda_{1}(L)$. 
Proof. Suppose that $f_{\alpha}$ is the first eigenfuction for the Laplace operator on $M_{\alpha}$ satisfying

$$
\int_{M_{\alpha}} f_{\alpha} d V=0, \quad \int_{M_{\alpha}} f_{\alpha}^{2} d V=1, \quad \text { and } \quad \int_{M_{\alpha}}\left|\nabla^{M_{\alpha}} f_{\alpha}\right|^{2} d V=\lambda_{1}\left(M_{\alpha}\right) .
$$

Because $\Delta_{M_{\alpha}} f_{\alpha}=-\lambda_{1}\left(M_{\alpha}\right) f_{\alpha}$, one has

$$
\Delta_{M_{\alpha}} f_{\alpha}^{2}=-2 \lambda_{1}\left(M_{\alpha}\right) f_{\alpha}^{2}+2\left|\nabla^{M_{\alpha}} f_{\alpha}\right|^{2} \geq-2 \lambda_{1}\left(M_{\alpha}\right) f_{\alpha}^{2}
$$

Assume that the theorem is not true. Then there exists a subsequence $\left\{\alpha_{j}\right\}$ which tends to zero, such that $\lambda_{1}\left(M_{\alpha_{j}}\right)<\frac{1}{4} \lambda_{1}(L)$. By Lemma 5', one has

$$
f_{\alpha_{j}}^{2} \leq C \int_{M_{\alpha}} f_{\alpha_{j}}^{2} d V \leq C
$$

Since $\lambda_{1}\left(M_{\alpha_{j}}\right)$ and $\operatorname{Vol}\left(M_{\alpha_{j}}\right)$ are bounded uniformly, the constant $C$ is independent of $j$.

Let $\varphi_{\delta}$ be a nonnegative function in $N$ satisfying $\varphi_{\delta} \equiv 1$ on $N \backslash B_{\delta}, \varphi_{\delta} \equiv 0$ on $B_{\frac{\delta}{2}}, \quad 0 \leq \varphi_{\delta} \leq 1$ on $B_{\delta} \backslash B_{\frac{\delta}{2}}$, and $\left|\nabla^{N} \varphi_{\delta}\right| \leq \frac{3}{\delta}$. A direct computation shows that

$$
\begin{aligned}
& \int_{M_{\alpha_{j}}}\left|\nabla^{M_{\alpha_{j}}} \varphi_{\delta} f_{\alpha_{j}}\right|^{2} d V \\
& =\int_{M_{\alpha_{j}}}\left(\left|\nabla^{M_{\alpha_{j}}} \varphi_{\delta}\right|^{2} f_{\alpha_{j}}^{2}+\varphi_{\delta}^{2}\left|\nabla^{M_{\alpha_{j}}} f_{\alpha_{j}}\right|^{2}+2 \varphi_{\delta} f_{\alpha_{j}} \nabla^{M_{\alpha_{j}} \varphi_{\delta}} \cdot \nabla^{M_{\alpha_{j}}} f_{\alpha_{j}}\right) d V \\
& \leq 2 \int_{M_{\alpha_{j}}} \varphi_{\delta}^{2}\left|\nabla^{M_{\alpha_{j}}} f_{\alpha_{j}}\right|^{2} d V+2 \int_{M_{\alpha_{j}}}\left|\nabla^{M_{\alpha_{j}}} \varphi_{\delta}\right|^{2} f_{\alpha_{j}}^{2} d V \\
& \leq 2 \int_{M_{\alpha_{j}}}\left|\nabla^{M_{\alpha_{j}}} f_{\alpha_{j}}\right|^{2} d V+2 \int_{M_{\alpha_{j}} \cap B_{\delta} \backslash B_{\frac{\delta}{2}}}\left|\nabla^{M_{\alpha_{j}}} \varphi_{\delta}\right|^{2} f_{\alpha_{j}}^{2} d V \\
& \leq 2 \lambda_{1}\left(M_{\alpha_{j}}\right)+\frac{C}{\delta^{2}} \operatorname{Vol}\left(M_{\alpha_{j}} \cap B_{\delta} \backslash B_{\frac{\delta}{2}}\right) \\
& \leq 2 \lambda_{1}\left(M_{\alpha_{j}}\right)+C \delta^{n-2} .
\end{aligned}
$$

We use $\left|\nabla^{M_{\alpha_{j}}} \varphi_{\delta}\right| \leq\left|\nabla^{N} \varphi_{\delta}\right|$ and $\operatorname{Vol}\left(M_{\alpha_{j}} \cap B_{\delta}\right) \leq C \delta^{n}$ in the above estimates. The area bound of $M_{\alpha_{j}} \cap B_{\delta}$ follows from the monotonicity formula for submanifolds with bounded mean curvature [21]. We also have

$$
\int_{M_{\alpha_{j}}}\left(\varphi_{\delta} f_{\alpha_{j}}\right)^{2} d V \geq 1-\int_{M_{\alpha_{j}} \cap B_{\delta}} f_{\alpha_{j}}^{2} d V \geq 1-C \operatorname{Vol}\left(M_{\alpha_{j}} \cap B_{\delta}\right) \geq 1-C \delta^{n}
$$


and

$$
\left(\int_{M_{\alpha_{j}}} \varphi_{\delta} f_{\alpha_{j}} d V\right)^{2}=\left(\int_{M_{\alpha_{j}} \cap B_{\delta}}\left(1-\varphi_{\delta}\right) f_{\alpha_{j}} d V\right)^{2} \leq C \operatorname{Vol}\left(M_{\alpha_{j}} \cap B_{\delta}\right)^{2} \leq C \delta^{2 n} .
$$

Recall that $M_{\alpha_{j}}$ is the same as $L$ in $N \backslash B_{\frac{\delta}{2}}$ for $\alpha_{j} \leq \frac{K \delta}{2}$. Therefore,

$$
\begin{gathered}
\frac{\int_{M_{\alpha_{j}}}\left|\nabla^{M_{\alpha_{j}}} \varphi_{\delta} f_{\alpha_{j}}\right|^{2} d V}{\int_{M_{\alpha_{j}}}\left(\varphi_{\delta} f_{\alpha_{j}}\right)^{2} d V-\operatorname{Vol}(L)^{-1}\left(\int_{M_{\alpha_{j}}} \varphi_{\delta} f_{\alpha_{j}} d V\right)^{2}} \\
=\frac{\int_{L}\left|\nabla^{L} \varphi_{\delta} f_{\alpha_{j}}\right|^{2} d V}{\int_{L}\left(\varphi_{\delta} f_{\alpha_{j}}\right)^{2} d V-\operatorname{Vol}(L)^{-1}\left(\int_{L} \varphi_{\delta} f_{\alpha_{j}} d V\right)^{2}} \geq \lambda_{1}(L) .
\end{gathered}
$$

On the other hand, it follows from the above estimates that

$$
\frac{\int_{M_{\alpha_{j}}}\left|\nabla^{M_{\alpha_{j}}} \varphi_{\delta} f_{\alpha_{j}}\right|^{2} d V}{\int_{M_{\alpha_{j}}}\left(\varphi_{\delta} f_{\alpha_{j}}\right)^{2} d V-\operatorname{Vol}(L)^{-1}\left(\int_{M_{\alpha_{j}}} \varphi_{\delta} f_{\alpha_{j}} d V\right)^{2}} \leq \frac{2 \lambda_{1}\left(M_{\alpha_{j}}\right)+C \delta^{n-2}}{1-C \delta^{2 n}} .
$$

Choose $\delta$ small enough so that $C \delta^{n-2}<\min \left(\frac{\lambda_{1}(L)}{4}, \frac{1}{4}\right)$. Then by combining the two inequalities, one gets $\lambda_{1}\left(M_{\alpha_{j}}\right)>\frac{1}{4} \lambda_{1}(L)$ when $\alpha_{j} \leq \frac{K \delta}{2}$, which is a contradiction. Thus the theorem is proved.

\section{Q.E.D.}

Remark: It is easy to see from the proof that the lower bound can be improved and the estimate also works for other singularities. Because the submanifold $L$ is closed and connected, its first eigenvalue $\lambda_{1}(L)$ is a positive number.

In the case $n=2$, one also can construct $M_{\alpha}$, but $\varepsilon$ may be chosen differently. We need to modify the function $\varphi_{\delta}$ in this situation as follows:

$$
\varphi_{\delta}(x)=\left\{\begin{array}{cl}
0 & |x|<\delta^{2} \\
\left(\frac{\log \frac{|x|}{\delta^{2}}}{\log \frac{1}{\delta}}\right) & \delta^{2} \leq|x| \leq \delta . \\
1 & |x|>\delta
\end{array}\right.
$$


A direct computation gives

$$
\int_{M_{\alpha_{j}} \cap B_{\delta} \backslash B_{\delta^{2}}}\left|\nabla^{M_{\alpha_{j}}} \varphi_{\delta}\right|^{2} d V \leq \frac{C}{|\log \delta|} .
$$

Recall that $M_{\alpha_{j}}$ is the same as $L$ in $N \backslash B_{\delta^{2}}$ for $\alpha_{j} \leq K \delta^{2}$. Similar arguments as in the case $n>2$ lead to

$$
\lambda_{1}(L) \leq \frac{2 \lambda_{1}\left(M_{\alpha_{j}}\right)+\frac{C}{|\log \delta|}}{1-C \delta^{4}} .
$$

Choose $\delta$ small enough so that $\frac{C}{|\log \delta|}<\frac{\lambda_{1}(L)}{4}$ and $C \delta^{4} \leq \frac{1}{4}$. One will get $\lambda_{1}\left(M_{\alpha_{j}}\right)>\frac{1}{4} \lambda_{1}(L)$ when $\alpha_{j} \leq K \delta^{2}$, which is a contradiction. Thus we obtain the same result.

\section{Perturbation.}

There exists a constant $c_{1}$ such that the exponential map from the normal bundle $T^{\perp} M_{\alpha}$ into $N$ is an embedding in the $c_{1} \varepsilon$ neighborhood of $M_{\alpha}$. Choose a smooth function $\eta_{\alpha}$ such that $\eta_{\alpha}(s) \equiv 1$ when $|s| \leq \frac{c_{1} \varepsilon}{2}$, and $\eta_{\alpha}(s) \equiv 0$ when $|s| \geq \frac{3 c_{1} \varepsilon}{4}$. Moreover, it also satisfies

$$
0 \leq \eta_{\alpha}(s) \leq 1, \quad\left|\nabla \eta_{\alpha}(s)\right| \leq \frac{C}{\varepsilon}, \quad\left|\nabla^{2} \eta_{\alpha}(s)\right| \leq \frac{C}{\varepsilon^{2}}, \quad \text { and } \quad\left|\nabla^{3} \eta_{\alpha}(s)\right| \leq \frac{C}{\varepsilon^{3}}
$$

for every $s$. Given a $C^{2, \beta}$ function $u$ on $M_{\alpha}, 0 \leq \beta \leq 1$, we can extend it into a $C^{2, \beta}$ function $U$ on $N$ by defining $U(\exp (x, v))=\eta_{\alpha}(|v|) u(x)$ for $x \in M_{\alpha}$ and $v \in T_{x}^{\perp} M_{\alpha}$. We then solve the Hamiltonian flow:

$$
\frac{\partial \varphi(t, q)}{\partial t}=-J \nabla^{N} U(\varphi(t, q)) \text { and } \varphi(0, q)=q \text { for } q \in N
$$

There exists a unique $C^{1, \beta}$ solution for small $t$. Note that if $\varphi_{U}(t, q)$ is a solution defined by $U$, then $\varphi_{U}(s t, q)$ is a solution defined by $s U$. Denote

$$
\varphi_{u}(x)=\varphi_{U}(1, x) \text { for } x \in M_{\alpha} .
$$

The map $\varphi_{u}$ can be defined for $u$ in a neighborhood of the zero function. In particular, it is defined when $\left\|\left(\nabla^{N}\right)^{2} U\right\|_{0, N}<1$. Because $\varphi_{U}(1, q)$ is a symplectic map, the image $\varphi_{u}\left(M_{\alpha}\right)$ is Lagrangian. Moreover, the family of maps $\varphi_{t u}, 0 \leq t \leq 1$, is a homotopy between $\varphi_{u}$ and the identity map $\varphi_{0}$. 
Define a $C^{0, \beta}$ function on $M_{\alpha}$ by $\mathcal{F}_{\alpha}(u)(x)=-\star \varphi_{u}^{*}(\operatorname{Im} \Omega)(x)$, where $\star$ is the Hodge star operator with respect to the induced metric on $M_{\alpha}$. If we can find a function $u$ such that $\varphi_{u}$ is an embedding and satisfies $\mathcal{F}_{\alpha}(u)=0$, then $\varphi_{u}\left(M_{\alpha}\right)$ will be an embedded special Lagrangian submanifold. Therefore, the goal is to find the zero set of $\mathcal{F}_{\alpha}$. The differential of $\mathcal{F}_{\alpha}$ at the zero function is

$$
D \mathcal{F}_{\alpha}(0)(u)=\star \varphi_{0}^{*}\left(d \iota_{J \nabla^{N} U} \operatorname{Im} \Omega\right)(x),
$$

where $\iota_{J \nabla^{N} U}$ is the interior derivative in $J \nabla^{N} U$ direction. Because $M_{\alpha}$ is Lagrangian, there exists a function $\theta(x)(\bmod 2 \pi)$ on $M_{\alpha}$, such that

$$
\left.\Omega\right|_{M_{\alpha}}=e^{i \theta(x)} \omega_{1} \wedge \cdots \wedge \omega_{n}
$$

where $\omega_{1} \cdots \omega_{n}$ is a local orthonormal basis on the cotangent bundle $T^{*} M_{\alpha}$ [19]. Note that

$$
\begin{aligned}
& \varphi_{0}^{*}\left(i_{J \nabla^{N} U} \operatorname{Im} \Omega\right) \\
=\operatorname{Im} \sum_{\beta=1}^{n} e^{i \theta(x)}[ & i\left(J \nabla^{N} U\right)^{n+\beta} \omega_{1} \wedge \cdots \wedge \stackrel{\vee}{\omega}_{\beta} \cdots \wedge \omega_{n} \\
& \left.+\left(J \nabla^{N} U\right)^{\beta} \omega_{1} \wedge \cdots \wedge \stackrel{\vee}{\omega}_{\beta} \cdots \wedge \omega_{n}\right] \\
= & \cos \theta(x) \star d u,
\end{aligned}
$$

where $\stackrel{\vee}{\omega}_{\beta}$ means that $\omega_{\beta}$ does not appear and the last equality follows from the fact that $U$ is constant along normal lines near $M_{\alpha}$. Because $H=J \nabla^{M_{\alpha}} \theta$ [19], we thus have

$$
D \mathcal{F}_{\alpha}(0)(u)=\cos \theta(x) \Delta_{M_{\alpha}} u-\sin \theta(x)<H, J \nabla^{M_{\alpha}} u>.
$$

It will be denoted by $\mathcal{L} u$ for simplicity. Because $|\sin \theta|=\left|\varphi_{0}^{*}(\operatorname{Im} \Omega)\right| \leq C \alpha$, it follows that $|\theta(x)| \leq C \alpha$. One then can show

Proposition 2. When $\alpha$ is small, the operator $\mathcal{L}$ is an elliptic operator and its kernel consists of the constant functions. Moreover, the first eigenvalue $\lambda_{1}\left(M_{\alpha}, \mathcal{L}\right)$ for the operator $\mathcal{L}$ on $M_{\alpha}$ has a uniform positive lower bound.

Proof. By choosing $\alpha$ small enough, we can assume that $\cos \theta(x)>\frac{1}{2}$. Hence $\mathcal{L}$ is an elliptic operator. Constants are clearly in the kernel of $\mathcal{L}$. Suppose that $\mathcal{L} u=0$. Multiply $u$ on both sides, and integrate over $M_{\alpha}$. We 
then get

$$
\begin{aligned}
0=\int_{M_{\alpha}} u \mathcal{L} u d V & =\int_{M_{\alpha}} u d(\cos \theta(x) \star d u) d V \\
& =-\int_{M_{\alpha}} d u \wedge \cos \theta(x) \star d u \\
& =-\int_{M_{\alpha}} \cos \theta|d u|^{2} d V \\
& \leq-\frac{1}{2} \int_{M_{\alpha}} \cos \theta|d u|^{2} d V
\end{aligned}
$$

It implies that $|d u|$ is identically zero. Thus the kernel of $\mathcal{L}$ consists of only constant solutions.

We now estimate $\lambda_{1}\left(M_{\alpha}, \mathcal{L}\right)$. Suppose that $f_{\alpha}$ is the first eigenfuction of $\mathcal{L}$, which satisfies

$$
\int_{M_{\alpha}} f_{\alpha} d V=0, \quad \int_{M_{\alpha}} f_{\alpha}^{2} d V=1, \quad \text { and } \quad \mathcal{L} f_{\alpha}=-\lambda_{1}\left(M_{\alpha}, \mathcal{L}\right) f_{\alpha}
$$

Multiply both sides of the equation by $-\frac{f_{\alpha}}{\cos \theta(x)}$ and integrate over $M_{\alpha}$. We have

$$
\begin{aligned}
& -\int_{M_{\alpha}} f_{\alpha} \Delta_{M_{\alpha}} f_{\alpha} d V+\int_{M_{\alpha}} f_{\alpha} \tan \theta(x)<H, J \nabla^{M_{\alpha}} f_{\alpha}>d V \\
= & \lambda_{1}\left(M_{\alpha}, \mathcal{L}\right) \int_{M_{\alpha}} \frac{f_{\alpha}^{2}}{\cos \theta(x)} d V .
\end{aligned}
$$

A direct computation shows that

$$
\begin{aligned}
& \left|\int_{M_{\alpha}} f_{\alpha} \tan \theta(x)<H, J \nabla^{M_{\alpha}} f_{\alpha}>d V\right| \\
\leq & C \max _{M_{\alpha}}(\tan \theta(x))\left(\int_{M_{\alpha}}\left|f_{\alpha}\right|^{2} d V\right)^{\frac{1}{2}}\left(\int_{M_{\alpha}}\left|\nabla^{M_{\alpha}} f_{\alpha}\right|^{2} d V\right)^{\frac{1}{2}} \\
\leq & C \max _{M_{\alpha}}(\tan \theta(x))\left(\int_{M_{\alpha}}\left|\nabla^{M_{\alpha}} f_{\alpha}\right|^{2} d V\right)^{\frac{1}{2}} .
\end{aligned}
$$


Plugging this into the above equality, we will get

$$
\begin{aligned}
& 2 \lambda_{1}\left(M_{\alpha}, \mathcal{L}\right) \\
\geq & \lambda_{1}\left(M_{\alpha}, \mathcal{L}\right) \int_{M_{\alpha}} \frac{f_{\alpha}^{2}}{\cos \theta(x)} d V \\
\geq & \int_{M_{\alpha}}\left|\nabla^{M_{\alpha}} f_{\alpha}\right|^{2} d V-C \max _{M_{\alpha}}(\tan \theta(x))\left(\int_{M_{\alpha}}\left|\nabla^{M_{\alpha}} f_{\alpha}\right|^{2} d V\right)^{\frac{1}{2}} \\
\geq & \frac{1}{2} \int_{M_{\alpha}}\left|\nabla^{M_{\alpha}} f_{\alpha}\right|^{2} d V \\
\geq & \frac{1}{2} \lambda_{1}\left(M_{\alpha}\right),
\end{aligned}
$$

when $\alpha$ is sufficiently small. This completes the proof of the proposition.

Q.E.D.

\section{The theorem.}

We first set some notation which will be used in the rest of this paper. Assume that $u$ is a function on $M_{\alpha}$. We denote

$$
\begin{gathered}
\|u\|_{0, M_{\alpha}}=\sup _{M_{\alpha}}|u|, \\
{[u]_{\beta, M_{\alpha}}=\sup _{x, x^{\prime} \in M_{\alpha}} \frac{\left|u(x)-u\left(x^{\prime}\right)\right|}{\operatorname{dist}\left(x, x^{\prime}\right)^{\beta}}, \quad 0<\beta<1,}
\end{gathered}
$$

and

$$
\|u\|_{L^{p}}=\left(\int_{M_{\alpha}} u^{p} d V\right)^{\frac{1}{p}} .
$$

We can embed $N$ isometrically into $R^{l}$ and set

$$
\left[\left(\nabla^{M_{\alpha}}\right)^{k} u\right]_{\beta, M_{\alpha}}=\sup _{x, x^{\prime} \in M_{\alpha}} \frac{\left|\left(\nabla^{M_{\alpha}}\right)^{k} u(x)-\left(\nabla^{M_{\alpha}}\right)^{k} u\left(x^{\prime}\right)\right|}{\operatorname{dist}\left(x, x^{\prime}\right)^{\beta}}
$$

where $k$ is a positive integer.

When $\alpha$ tends to zero, the neck on $M_{\alpha}$ will shrink to $p$. Thus we need to introduce a weighted norm to do the estimates. Roughly speaking, we want to choose the weight function $\rho(x)$ on $M_{\alpha}$ such that $\rho(x)$ is less than the 
radius of a normal ball at $x \in M_{\alpha}$. More precisely, we can choose that $\rho(x)$ is of the form [2]:

$$
\rho(x)= \begin{cases}c \varepsilon & \text { for } x \in M_{\alpha} \cap B_{\varepsilon r_{2}} \\ \text { interpolation } & \text { for } x \in M_{\alpha} \cap B_{r_{2}} \backslash B_{\varepsilon r_{2}} \\ R_{2} & \text { for } x \in M_{\alpha} \backslash B_{r_{2}}\end{cases}
$$

for some constants $r_{2}$ and $R_{2}$. In addition, we can also require $\rho(x)$ to satisfy the following properties:

1. $\left\|\nabla^{M_{\alpha}} \rho\right\|_{0, M_{\alpha}} \leq C$,

2. $c \alpha \leq \rho(x) \leq C \alpha$ for $x \in T_{1} \cup T_{2}$,

3. $\left\|\rho^{-1}\right\|_{L^{p}} \leq C$ for $p<n$.

Definition 3. Let $u$ be a $C^{k, \beta}$ function on $M_{\alpha}$, where $k$ is an integer and $0<\beta<1$. The $\rho$-weighted $(k, \beta)$ norm $\|u\|_{C_{\rho}^{k, \beta}\left(M_{\alpha}\right)}$ of $u$ is defined as the sum:

$\|u\|_{0, M_{\alpha}}+\left\|\rho\left|\nabla^{M_{\alpha}} u\right|\right\|_{0, M_{\alpha}}+\cdots+\left\|\rho^{k}\left|\left(\nabla^{M_{\alpha}}\right)^{k} u\right|\right\|_{0, M_{\alpha}}+\left[\rho^{k+\beta}\left(\nabla^{M_{\alpha}}\right)^{k} u\right]_{\beta, M_{\alpha}}$.

Proposition 3. The operator $\mathcal{L}$ is a bounded operator between the Banach space $C^{2, \beta}\left(M_{\alpha}\right)$ with norm $\|\cdot\|_{C_{\rho}^{2, \beta}\left(M_{\alpha}\right)}$ and the Banach space $C^{0, \beta}\left(M_{\alpha}\right)$ with norm $\left\|\rho^{2} \cdot\right\|_{C_{\rho}^{0, \beta}\left(M_{\alpha}\right)}$.

Proof. Note that

$$
\begin{aligned}
& \left\|\rho^{2} \mathcal{L} u\right\|_{C_{\rho}^{0, \beta}\left(M_{\alpha}\right)} \\
\leq & \left\|\rho^{2} \cos \theta \Delta_{M_{\alpha}} u\right\|_{C_{\rho}^{0, \beta}\left(M_{\alpha}\right)}+\left\|\rho^{2} \sin \theta<H, J \nabla^{M_{\alpha}} u>\right\|_{C_{\rho}^{0, \beta}\left(M_{\alpha}\right)} .
\end{aligned}
$$

A direct computation gives

$$
\begin{aligned}
& \left\|\rho^{2} \cos \theta \Delta_{M_{\alpha}} u\right\|_{C_{\rho}^{0, \beta}\left(M_{\alpha}\right)} \\
\leq & \left\|\rho^{2} \Delta_{M_{\alpha}} u\right\|_{0, M_{\alpha}}+\left[\rho^{2+\beta} \Delta_{M_{\alpha}} u\right]_{\beta, M_{\alpha}}+[\cos \theta]_{\beta, M_{\alpha}}\left\|\rho^{\beta}\right\|_{0, M_{\alpha}}\left\|\rho^{2} \Delta_{M_{\alpha}} u\right\|_{0, M_{\alpha}} \\
\leq & C\|u\|_{C_{\rho}^{2, \beta}\left(M_{\alpha}\right)} .
\end{aligned}
$$


We also have

$$
\begin{aligned}
& \left\|\rho^{2} \sin \theta<H, J \nabla^{M_{\alpha}} u>\right\|_{C_{\rho}^{0, \beta}\left(M_{\alpha}\right)} \\
\leq & \|\rho \sin \theta|H|\|_{0, M_{\alpha}}\left\|\rho\left|\nabla^{M_{\alpha}} u\right|\right\|_{0, M_{\alpha}}+\|\rho \sin \theta|H|\|_{0, M_{\alpha}}\left[\rho^{1+\beta} \nabla^{M_{\alpha}} u\right]_{\beta, M_{\alpha}} \\
& +[\rho \sin \theta H]_{\beta, M_{\alpha}}\left\|\rho^{\beta}\right\|_{0, M_{\alpha}}\left\|\rho\left|\nabla^{M_{\alpha}} u\right|\right\|_{0, M_{\alpha}} .
\end{aligned}
$$

Using the fact that the mean curvature is zero outside a small ball $B_{r}(p)^{\prime}$ and the properties of $\rho$ and $\sin \theta$, it follows that

$$
\left\|\rho^{2} \sin \theta<H, J \nabla^{M_{\alpha}} u>\right\|_{0, M_{\alpha}} \leq C \alpha^{2}\left\|\rho\left|\nabla^{M_{\alpha}} u\right|\right\|_{0, M_{\alpha}} .
$$

Moreover, all the sup. norm involved are taken over $B_{r}(p)^{\prime}$ when we estimate $\left\|\rho^{2} \sin \theta<H, J \nabla^{M_{\alpha}} u>\right\|_{C_{\rho}^{0, \beta}\left(M_{\alpha}\right)}$. When $\operatorname{dist}\left(x, x^{\prime}\right) \geq \alpha$, one has that

$$
\frac{\left|\rho \sin \theta H(x)-\rho \sin \theta H\left(x^{\prime}\right)\right|}{\operatorname{dist}\left(x, x^{\prime}\right)^{\beta}} \leq C \alpha^{2-\beta} .
$$

When $\operatorname{dist}\left(x, x^{\prime}\right) \leq \alpha$, one has

$$
\frac{\left|\rho \sin \theta H(x)-\rho \sin \theta H\left(x^{\prime}\right)\right|}{\operatorname{dist}\left(x, x^{\prime}\right)^{\beta}} \leq C \alpha \alpha^{1-\beta}=C \alpha^{2-\beta} .
$$

Here we use $\left\|\left(\nabla^{M_{\alpha}}\right)^{2} \sin \theta\right\|_{0, M_{\alpha}} \leq C \alpha^{-1}$ to get estimates involved $H$. The upshot is

$$
\left\|\rho^{2} \sin \theta<H, J \nabla^{M_{\alpha}} u>\right\|_{C_{\rho}^{0, \beta}\left(M_{\alpha}\right)} \leq C \alpha^{2-\beta}\|u\|_{C_{\rho}^{1, \beta}\left(M_{\alpha}\right)}
$$

and hence

$$
\left\|\rho^{2} \mathcal{L} u\right\|_{C_{\rho}^{0, \beta}\left(M_{\alpha}\right)} \leq C\|u\|_{C_{\rho}^{2, \beta}\left(M_{\alpha}\right)}
$$

Q.E.D.

By elliptic Schauder estimates [2] for $\rho$-weighted $(k, \beta)$ norms, one can prove that

$$
\|u\|_{C_{\rho}^{2, \beta}\left(M_{\alpha}\right)} \leq C \varepsilon^{-\beta}\left(\left\|\rho^{2} \Delta_{M_{\alpha}} u\right\|_{C_{\rho}^{0, \beta}\left(M_{\alpha}\right)}+\|u\|_{0, M_{\alpha}}\right) .
$$

We show in Appendix that $\|u\|_{0, M_{\alpha}} \leq C \varepsilon^{-\nu}\left\|\rho^{2} \Delta_{M_{\alpha}} u\right\|_{C_{\rho}^{0, \beta}\left(M_{\alpha}\right)}$ for $u$ satisfying $\int_{M_{\alpha}} u d V=0$. We thus have

$$
\|u\|_{C_{\rho}^{2, \beta}\left(M_{\alpha}\right)} \leq C \varepsilon^{-(\beta+\nu)}\left\|\rho^{2} \Delta_{M_{\alpha}} u\right\|_{C_{\rho}^{0, \beta}\left(M_{\alpha}\right)} .
$$

In next Lemma, we bound $\left\|\rho^{2} \Delta_{M_{\alpha}} u\right\|_{C_{\rho}^{0, \beta}\left(M_{\alpha}\right)}$ by $\left\|\rho^{2} \mathcal{L} u\right\|_{C_{\rho}^{0, \beta}\left(M_{\alpha}\right)}$ and prove 
Lemma 2. For any given positive number $\nu$, there exists $\alpha_{0}$ such that the following estimate holds. Suppose that $\alpha<\alpha_{0}$ and $u$ is a $C^{2, \beta}$ function on $M_{\alpha}$, which satisfies $\int_{M_{\alpha}} u d V=0$. Then

$$
\|u\|_{C_{\rho}^{2, \beta}\left(M_{\alpha}\right)} \leq C \varepsilon^{-(\beta+\nu)}\left\|\rho^{2} \mathcal{L} u\right\|_{C_{\rho}^{0, \beta}\left(M_{\alpha}\right)},
$$

where the constant $C$ depends on $\nu$, but is independent of $\alpha$.

Proof. Note that

$\left\|\rho^{2} \mathcal{L} u\right\|_{C_{\rho}^{0, \beta}\left(M_{\alpha}\right)} \geq\left\|\rho^{2} \cos \theta \Delta_{M_{\alpha}} u\right\|_{C_{\rho}^{0, \beta}\left(M_{\alpha}\right)}-\left\|\rho^{2} \sin \theta<H, J \nabla^{M_{\alpha}} u>\right\|_{C_{\rho}^{0, \beta}\left(M_{\alpha}\right)}$,

and

$$
\left\|\rho^{2} \cos \theta \Delta_{M_{\alpha}} u\right\|_{C_{\rho}^{0, \beta}\left(M_{\alpha}\right)}=\left\|\rho^{2} \cos \theta \Delta_{M_{\alpha}} u\right\|_{0, M_{\alpha}}+\left[\rho^{2+\beta} \cos \theta \Delta_{M_{\alpha}} u\right]_{\beta, M_{\alpha}} .
$$

When $\alpha$ is small, we have

$$
\left\|\rho^{2} \cos \theta \Delta_{M_{\alpha}} u\right\|_{0, M_{\alpha}} \geq \frac{1}{2}\left\|\rho^{2} \Delta_{M_{\alpha}} u\right\|_{0, M_{\alpha}}
$$

and

$$
\left[\rho^{2+\beta} \cos \theta \Delta_{M_{\alpha}} u\right]_{\beta, M_{\alpha}} \geq \frac{1}{2}\left[\rho^{2+\beta} \Delta_{M_{\alpha}} u\right]_{\beta, M_{\alpha}}-C \alpha\left\|\rho^{2} \Delta_{M_{\alpha}} u\right\|_{0, M_{\alpha}} .
$$

Hence

$$
\left\|\rho^{2} \cos \theta \Delta_{M_{\alpha}} u\right\|_{C_{\rho}^{0, \beta}\left(M_{\alpha}\right)} \geq \frac{1}{3}\left\|\rho^{2} \Delta_{M_{\alpha}} u\right\|_{C_{\rho}^{0, \beta}\left(M_{\alpha}\right)} .
$$

On the other hand, we have

$$
\begin{aligned}
\left\|\rho^{2} \sin \theta<H, J \nabla^{M_{\alpha}} u>\right\|_{C_{\rho}^{0, \beta}\left(M_{\alpha}\right)} & \leq C \alpha^{2-\beta}\|u\|_{C_{\rho}^{1, \beta}\left(M_{\alpha}\right)} \\
& \leq C \alpha^{2-\beta} \varepsilon^{-(\beta+\nu)}\left\|\rho^{2} \Delta_{M_{\alpha}} u\right\|_{C_{\rho}^{0, \beta}\left(M_{\alpha}\right)} .
\end{aligned}
$$

Putting all these estimates together, we get

$$
\left\|\rho^{2} \mathcal{L} u\right\|_{C_{\rho}^{0, \beta}\left(M_{\alpha}\right)} \geq \frac{1}{4}\left\|\rho^{2} \Delta_{M_{\alpha}} u\right\|_{C_{\rho}^{0, \beta}\left(M_{\alpha}\right)}
$$

and the proposition is therefore proved.

\section{Q.E.D.}


Denote the Banach space of $C^{2, \beta}$ functions on $M_{\alpha}$ which satisfies $\int_{M_{\alpha}} u d V=$ 0 with norm $\|\cdot\|_{C_{\rho}^{2, \beta}\left(M_{\alpha}\right)}$ by $\mathcal{B}_{1}$ and the Banach space of $C^{0, \beta}$ functions on $M_{\alpha}$ which satisfies $\int_{M_{\alpha}} u d V=0$ with norm $\left\|\rho^{2} \cdot\right\|_{C_{\rho}^{0, \beta}\left(M_{\alpha}\right)}$ by $\mathcal{B}_{2}$. Because $\int_{M_{\alpha}} \operatorname{Im} \Omega=0$ and the family of maps $\varphi_{t u}, 0 \leq t \leq 1$, is a homotopy between $\varphi_{u}$ and $\varphi_{0}$, it follows that $\int_{M_{\alpha}} \mathcal{F}_{\alpha}(u) d V=0$. Thus we can restrict $\mathcal{F}_{\alpha}$ as a map from $\mathcal{B}_{1}$ into $\mathcal{B}_{2}$. A direct computation shows that the operator $\mathcal{L}$ is self-adjoint. By Proposition 2, we consequently have:

Proposition 4. The operator $\mathcal{L}$ from $\mathcal{B}_{1}$ into $\mathcal{B}_{2}$ is injective and surjective.

We will apply the following version of inverse function theorem to $\mathcal{F}_{\alpha}$.

Theorem 2. [1] Let $\mathcal{F}: \mathcal{B} \rightarrow \mathcal{B}^{\prime}$ be a $C^{1}$ map between Banach spaces and suppose that the differential $D \mathcal{F}(0)$ of $\mathcal{F}$ at 0 is an isomorphism. Moreover, suppose that $\mathcal{F}$ satisfies the estimates:

1. $\|D \mathcal{F}(0) x\|_{\mathcal{B}^{\prime}} \geq C_{L}\|x\|_{\mathcal{B}}$ for any $x \in \mathcal{B}$,

2. $\|D \mathcal{F}(0) y-D \mathcal{F}(x) y\|_{\mathcal{B}^{\prime}} \leq C_{N}\|x\|_{\mathcal{B}}\|y\|_{\mathcal{B}}$ for all $x$ sufficiently near 0 and for any $y \in \mathcal{B}$,

where $C_{L}$ and $C_{N}$ are constants independent of $x$ and $y$. Then there exist neighborhoods $\mathcal{U}$ of 0 and $\mathcal{V}$ of $\mathcal{F}(0)$ so that $\mathcal{F}: \mathcal{U} \rightarrow \mathcal{V}$ is a $C^{1}$ diffeomorphism and $\mathcal{V}$ contains the ball $B_{\frac{C_{L}}{2} r}(\mathcal{F}(0))$, where $r \leq \frac{C_{L}}{2 C_{N}}$. Furthermore, the image of the ball $B_{r}(0)$ under $\mathcal{F}$ contains the ball $B_{\frac{C_{L}}{2} r}(\mathcal{F}(0))$.

We already get an estimate on $C_{L}$ in Lemma 2 and still need an estimate on $C_{N}$ to apply Theorem 2 .

Lemma 3. Assume that $v \in \mathcal{B}_{1}$ and is sufficiently close to 0 . Then the differential of $\mathcal{F}_{\alpha}$ at $v$ satisfies the following estimate:

$$
\left\|\rho^{2}\left(D \mathcal{F}_{\alpha}(v)(u)-D \mathcal{F}_{\alpha}(0)(u)\right)\right\|_{C_{\rho}^{0, \beta}\left(M_{\alpha}\right)} \leq C \varepsilon^{-2}\|v\|_{C_{\rho}^{2, \beta}\left(M_{\alpha}\right)}\|u\|_{C_{\rho}^{2, \beta}\left(M_{\alpha}\right)}
$$

for all $u \in \mathcal{B}_{1}$.

Proof. Suppose that $g$ is the Ricci flat metric on $N$. For a given constant $s>0$, we can define a conformal metric $g_{s}=s^{-2} g$. Let $w$ be a $C^{2, \beta}$ function on $N$ and define $w_{s}=s^{-2} w$. Then the Hamiltonian flow $\varphi_{w}$ determined by $w$ in the metric $g$ is the same as the Hamiltonian flow $\varphi_{w_{s}}^{s}$ determined by $w_{s}$ in the metric $g_{s}$. The form $\Omega_{s}=s^{-n} \Omega$ is a holomorphic $(n, 0)$ form of 
unit length in the metric $g_{s}$. Define $G_{\alpha}^{s}\left(w_{s}\right)=\star_{s}\left(\varphi_{w_{s}}^{s}\right)^{*}\left(\operatorname{Im} \Omega_{s}\right)$, where $\star_{s}$ is the Hodge star operator with respect to the metric on $M_{\alpha}$ induced from $g_{s}$. Since

$$
\begin{aligned}
\star_{s}\left(\varphi_{w_{s}}^{s}\right) *\left(\operatorname{Im} \Omega_{s}\right) & =\star_{s}\left(\varphi_{w_{s}}^{s}\right)^{*}\left(s^{-n} \operatorname{Im} \Omega\right) \\
& =\star_{s} s^{-n} \varphi_{w}^{*}(\operatorname{Im} \Omega) \\
& =\star \varphi_{w}^{*}(\operatorname{Im} \Omega)
\end{aligned}
$$

thus $G_{\alpha}^{s}\left(w_{s}\right)=\mathcal{F}_{\alpha}(w)$. Assume that $K_{1} \geq \frac{1}{2}$ is an upper bound of $\left\|\nabla^{M_{\alpha}} \rho\right\|_{0, M_{\alpha}}$. Choose $x \in M_{\alpha}$ and let $s=\rho(x)$. Then the function $\rho$ satisfies $\frac{s}{2} \leq \rho \leq \frac{3 s}{2}$ in the ball $B_{\frac{s}{2 K_{1}}(x)}$ and the induced metric are bounded uniformly in this ball. Fix this $s$ and denote $g_{s}$ by $g^{\prime}$ from now on. Denote the norm with respect to the metric $g^{\prime}$ by $\|\cdot\|^{g^{\prime}}$. We have

$$
\begin{aligned}
& \left\|\rho^{2}\left(\left.\frac{d \mathcal{F}_{\alpha}(v+t u)}{d t}\right|_{t=0}-\left.\frac{d \mathcal{F}_{\alpha}(t u)}{d t}\right|_{t=0}\right)\right\|_{0, B \frac{s}{2 K_{1}}}(x) \\
\leq & \frac{9 s^{2}}{4}\left\|D \mathcal{F}_{\alpha}(v)(u)-D \mathcal{F}_{\alpha}(0)(u)\right\|_{0, B \frac{s}{2 K_{1}}}(x) \\
= & \frac{9 s^{2}}{4}\left\|D G_{\alpha}^{s}\left(v_{s}\right)\left(u_{s}\right)-D G_{\alpha}^{s}(0)\left(u_{s}\right)\right\|_{0, B}^{g^{\prime}} \frac{1}{2 K_{1}}(x) \\
\leq & C s^{2}\left\|u_{s}\right\|_{C^{2}\left(B \frac{1}{2 K_{1}}(x)\right)}^{g^{\prime}}\left\|v_{s}\right\|_{C^{2}\left(B \frac{1}{2 K_{1}}(x)\right)}^{g^{\prime}},
\end{aligned}
$$

where in the last inequality we use the fact that $M_{\alpha}$ with the metric induced from $\left(N, g^{\prime}\right)$ has uniformly bounded geometry in $B_{\frac{1}{2 K_{1}}}(x)$. Because

$$
\begin{aligned}
& \left\|u_{s}\right\|_{C^{2}\left(B \frac{1}{2 K_{1}}(x)\right)}^{g^{\prime}} \\
= & \left\|s^{-2} u\right\|_{0, B \frac{1}{2 K_{1}}(x)}^{g^{\prime}}+\left\|\nabla^{g^{\prime}} s^{-2} u\right\|_{0, B \frac{1}{2 K_{1}}}^{g^{\prime}}(x) \\
= & \left.s^{-2}\|u\|_{0, B \frac{s}{2 K_{1}}}(x)+s^{-1}\|\nabla u\|_{0, B \frac{s}{2 K_{1}}}(x)+\| \nabla^{g^{\prime}}\right)^{2} s^{-2} u \|_{0, B \frac{s}{2 K_{1}}}^{g^{\prime}}(x) \\
\leq & C s^{-2}\|u\|_{C_{\rho}^{2}\left(M_{\alpha}\right)},
\end{aligned}
$$

it follows that

$$
\left\|\rho^{2}\left(D \mathcal{F}_{\alpha}(v)(u)-D \mathcal{F}_{\alpha}(0)(u)\right)\right\|_{0, B_{\frac{s}{2 K_{1}}}(x)} \leq C s^{-2}\|v\|_{C_{\rho}^{2}\left(M_{\alpha}\right)}\|u\|_{C_{\rho}^{2}\left(M_{\alpha}\right)} .
$$


We also need to estimate the following quantity (A):

$$
\frac{\left|\rho^{2+\beta}\left(D \mathcal{F}_{\alpha}(v)(u)-D \mathcal{F}_{\alpha}(0)(u)\right)(x)-\rho^{2+\beta}\left(D \mathcal{F}_{\alpha}(v)(u)-D \mathcal{F}_{\alpha}(0)(u)\right)\left(x^{\prime}\right)\right|}{\operatorname{dist}\left(x, x^{\prime}\right)^{\beta}} .
$$

When $\operatorname{dist}\left(x, x^{\prime}\right) \leq \frac{s}{2 K_{1}}$, we have

$$
\begin{aligned}
& (A) \leq \frac{\left|\rho^{2+\beta}(x)-\rho^{2+\beta}\left(x^{\prime}\right)\right|}{\operatorname{dist}\left(x, x^{\prime}\right)^{\beta}}\left\|D \mathcal{F}_{\alpha}(v)(u)-D \mathcal{F}_{\alpha}(0)(u)\right\|_{0, B_{\frac{s}{2 K_{1}}}}(x) \\
& +\rho^{2+\beta}\left(x^{\prime}\right)\left\|D \mathcal{F}_{\alpha}(v)(u)-D \mathcal{F}_{\alpha}(0)(u)\right\|_{\beta, B \frac{s}{2 K_{1}}}(x) \\
& \leq C s^{2}\left\|u_{s}\right\|_{C^{2, \beta}\left(B \frac{1}{2 K_{1}}(x)\right)}^{g^{\prime}}\left\|v_{s}\right\|_{C^{2, \beta}\left(B \frac{1}{2 K_{1}}(x)\right)}^{g^{\prime}} .
\end{aligned}
$$

Since

$$
\begin{aligned}
\left\|u_{s}\right\|_{C^{2, \beta}\left(B \frac{1}{2 K_{1}}(x)\right)}^{g^{\prime}} \leq s^{-2} & \|u\|_{0, B \frac{s}{2 K_{1}}(x)}+s\|\nabla u\|_{0, B} \frac{s}{2 K_{1}}(x) \\
& \left.+s^{2}\left\|\nabla^{2} u\right\|_{0, B \frac{s}{2 K_{1}}(x)}+s^{2+\beta}\left[\nabla^{2} u\right]_{\beta, B \frac{s}{2 K_{1}}(x)}\right)
\end{aligned}
$$

and

$$
\begin{aligned}
& \frac{s^{2+\beta}\left|\nabla^{2} u(x)-\nabla^{2} u\left(x^{\prime}\right)\right|}{\operatorname{dist}\left(x, x^{\prime}\right)^{\beta}} \\
\leq & C \frac{\left|\rho^{2+\beta}(x) \nabla^{2} u(x)-\rho^{2+\beta}(x) \nabla^{2} u\left(x^{\prime}\right)\right|}{\operatorname{dist}\left(x, x^{\prime}\right)^{\beta}} \\
\leq & C \frac{\left|\rho^{2+\beta}(x) \nabla^{2} u(x)-\rho^{2+\beta}\left(x^{\prime}\right) \nabla^{2} u\left(x^{\prime}\right)+\rho^{2+\beta}\left(x^{\prime}\right) \nabla^{2} u\left(x^{\prime}\right)-\rho^{2+\beta}(x) \nabla^{2} u\left(x^{\prime}\right)\right|}{\operatorname{dist}\left(x, x^{\prime}\right)^{\beta}} \\
\leq & C\left(\left[\rho^{2+\beta} \nabla^{2} u\right]_{\beta, B \frac{s}{2 K_{1}}(x)}+s^{2}\left\|\nabla^{2} u\right\|_{0, B} \frac{s}{2 K_{1}}(x)\right),
\end{aligned}
$$

one concludes that

$$
\left\|u_{s}\right\|_{C^{2, \beta}\left(B \frac{1}{2 K_{1}}(x)\right)}^{g^{\prime}} \leq C s^{-2}\|u\|_{C_{\rho}^{2, \beta}\left(M_{\alpha}\right)} .
$$

That is when $\operatorname{dist}\left(x, x^{\prime}\right) \leq \frac{s}{2 K_{1}}$, we have

$$
A \leq C s^{-2}\|v\|_{C_{\rho}^{2, \beta}\left(M_{\alpha}\right)}\|u\|_{C_{\rho}^{2, \beta}\left(M_{\alpha}\right)} .
$$


When $\operatorname{dist}\left(x, x^{\prime}\right) \geq \frac{s}{2 K_{1}}$, we also have

$$
\begin{aligned}
(A) & \left.\leq C s^{-\beta} s^{2+\beta}\left\|u_{s}\right\|_{C^{2}\left(B \frac{1}{2 K_{1}}(x)\right)}^{g^{\prime}}\left\|v_{s}\right\|_{C^{2}\left(B \frac{1}{2 K_{1}}\right.}^{g^{\prime}}(x)\right) \\
& \leq C s^{-2}\|v\|_{C_{\rho}^{2}\left(M_{\alpha}\right)}\|u\|_{C_{\rho}^{2}\left(M_{\alpha}\right)} .
\end{aligned}
$$

Putting all estimates together, we therefore get

$$
\left\|\rho^{2+\beta}\left(D \mathcal{F}_{\alpha}(v)(u)-D \mathcal{F}_{\alpha}(0)(u)\right)\right\|_{\beta, M_{\alpha}} \leq C \varepsilon^{-2}\|v\|_{C_{\rho}^{2, \beta}\left(M_{\alpha}\right)}\|u\|_{C_{\rho}^{2, \beta}\left(M_{\alpha}\right)} .
$$

That is,

$$
\left\|\rho^{2}\left(D \mathcal{F}_{\alpha}(v)(u)-D \mathcal{F}_{\alpha}(0)(u)\right)\right\|_{C_{\rho}^{0, \beta}\left(M_{\alpha}\right)} \leq C \varepsilon^{-2}\|v\|_{C_{\rho}^{2, \beta}\left(M_{\alpha}\right)}\|u\|_{C_{\rho}^{2, \beta}\left(M_{\alpha}\right)} .
$$

\section{Q.E.D.}

We can choose $\nu=\beta$ in Lemma 6 . Then choose $C_{L}=\frac{1}{C} \varepsilon^{2 \beta}$ by Lemma 2 and $C_{N}=C \varepsilon^{-2}$ by Lemma 3. Applying Theorem 2, we therefore conclude that the image of the ball $B_{r}(0)$ under $\mathcal{F}_{\alpha}$ contains the ball $B_{\frac{C_{L}}{2} r}\left(\mathcal{F}_{\alpha}(0)\right)$, where $r \leq \frac{\varepsilon^{2+2 \beta}}{2 C^{2}}$.

Lemma 4. When $n \geq 3$, the zero function lies in the ball $B_{\frac{C_{L}}{2} \varepsilon^{2+3 \beta}}\left(\mathcal{F}_{\alpha}(0)\right)$.

Proof. Denote $E=-\star \varphi_{0}^{*}(\operatorname{Im} \Omega)=\mathcal{F}_{\alpha}(0)$. Recall that $|E(x)| \leq C \alpha$ and $E(x)=0$ for $x \in M_{\alpha} \backslash B_{\frac{\alpha}{K}}$. This together with the properties of $\rho$ implies

$$
\left\|\rho^{2} E\right\|_{0, M_{\alpha}} \leq C \alpha^{3} \text {. }
$$

Moreover, we have

$$
\left|\nabla^{M_{\alpha}} E\right|=\left|\cos \theta \nabla^{M_{\alpha}} \theta\right| \leq|H| \leq C .
$$

When $\operatorname{dist}\left(x, x^{\prime}\right) \leq \alpha$, it follows that

$$
\frac{\left|\rho^{2+\beta} E(x)-\rho^{2+\beta} E\left(x^{\prime}\right)\right|}{\operatorname{dist}\left(x, x^{\prime}\right)^{\beta}} \leq C \alpha^{2+\beta} \alpha^{1-\beta} .
$$

When $\operatorname{dist}\left(x, x^{\prime}\right) \geq \alpha$, it follows that

$$
\frac{\left|\rho^{2+\beta} E(x)-\rho^{2+\beta} E\left(x^{\prime}\right)\right|}{\operatorname{dist}\left(x, x^{\prime}\right)^{\beta}} \leq C \alpha^{3+\beta} \alpha^{-\beta} .
$$

Since $\varepsilon=\alpha^{\frac{n+1}{n}}$ and $n \geq 3$, we thus have

$$
\left\|\rho^{2} E\right\|_{C_{\rho}^{0, \beta}\left(M_{\alpha}\right)} \leq C \alpha^{3} \leq \frac{C_{L}}{2} \varepsilon^{2+3 \beta}
$$

when $\beta$ and $\varepsilon$ are small enough. 
Q.E.D.

The extension function $U$ satisfies

$$
\begin{gathered}
\left\|\nabla^{N} U\right\|_{0, N} \leq C \varepsilon^{-1}\left(\|u\|_{0, M_{\alpha}}+\varepsilon\left\|\nabla^{M_{\alpha}} u\right\|_{0, M_{\alpha}}\right) \leq C \varepsilon^{-1}\|u\|_{C_{\rho}^{1}\left(M_{\alpha}\right)}, \\
\left\|\left(\nabla^{N}\right)^{2} U\right\|_{0, N} \leq C \varepsilon^{-2}\|u\|_{C_{\rho}^{2}\left(M_{\alpha}\right)} .
\end{gathered}
$$

When $\left\|\left(\nabla^{N}\right)^{2} U\right\|_{0, N} \leq \frac{1}{2}$, or $\|u\|_{C_{\rho}^{2}\left(M_{\alpha}\right)} \leq \frac{\varepsilon^{2}}{2 C}$, the map $\varphi_{u}$ is defined. When $\left\|\nabla^{N} U\right\|_{0, N} \leq c_{1} \varepsilon$, or $\|u\|_{C_{\rho}^{1}\left(M_{\alpha}\right)} \leq \frac{c_{1} \varepsilon^{2}}{C}$, the image $\varphi_{u}\left(M_{\alpha}\right)$ is embedded in a $c_{1} \varepsilon$ neighborhood of $M_{\alpha}$. Choose $r=\varepsilon^{2+3 \beta} \leq \frac{\varepsilon^{2+2 \beta}}{2 C^{2}}$ in Theorem 2. Then there exists a function $u \in \mathcal{B}_{1}$ with $\|u\|_{C_{\rho}^{2, \beta}\left(M_{\alpha}\right)} \leq \varepsilon^{2+3 \beta}$ such that $\mathcal{F}_{\alpha}(u)=0$. It follows that $\varphi_{u}\left(M_{\alpha}\right)$ is an embedded special Lagrangian submanifold. We hence prove the main theorem of the paper:

Theorem 3. Suppose that $L$ is a closed, connected, and immersed special Lagrangian submanifold in a closed Calabi-Yau manifold $N$ of complex dimension 3. Assume that $L$ has only isolated transversal self-intersection points. Then $L$ is the limit of a family of embedded closed special Lagrangian submanifolds in $N$.

Theorem 4. Suppose that $L$ is a closed, connected, and immersed special Lagrangian submanifold in a closed Calabi-Yau manifold $N$ of complex dimension $n>3$. Moreover, assume that $L$ has only isolated transversal selfintersection of two sheets and the two tangent planes at each intersection point satisfy the angle condition $\theta_{1}+\cdots+\theta_{n}=\frac{\pi}{2}$ (see section 2). Then $L$

is the limit of a family of embedded closed special Lagrangian submanifolds in $N$.

\section{Appendix : Sup. Norm Estimate.}

The De Giorgi-Nash estimates in this Appendix are based on [4], [18] and [22]. We modify the arguments to fit in the situation discussed in this paper and present the material here just for the reader's reference and completeness.

For a submanifold $M^{n} \subset R^{l}$, J. H. Michael and L. Simon [14] proved the Sobolev inequality:

$$
\left(\int_{M} h^{\frac{n}{n-1}} d V\right)^{\frac{n-1}{n}} \leq C(n) \int_{M}\left(\left|\nabla^{M} h\right|+h|\bar{H}|\right) d V
$$


where $h$ is a $C^{1}$ nonnegative function on $M$ with compact support and $\bar{H}$ is the mean curvature of $M$ in $R^{l}$. When $n>2$, the inequality can be converted easily into

$$
\left(\int_{M} h^{\frac{2 n}{n-2}} d V\right)^{\frac{n-2}{n}} \leq C(n)\left(\int_{M}\left|\nabla^{M} h\right|^{2} d V+\int_{M} h^{2}|\bar{H}|^{2} d V\right) .
$$

Or write in a scaling invariant form

$$
\left(\int_{M} h^{\frac{2 n}{n-2}} d V\right)^{\frac{n-2}{n}} \leq C(n)\left(\int_{M}\left|\nabla^{M} h\right|^{2} d V+\operatorname{Vol}(M)^{-\frac{2}{n}} \int_{M} h^{2} d V\right)
$$

The constant $C(n)$ also depends on $\sup |H|^{2} \operatorname{Vol}(M)^{\frac{2}{n}}$. But because both $\bar{H}$ and $\operatorname{Vol}(M)$ are uniformly bounded in our cases, we omit this dependency. When $n=2$, the Sobolev inequality implies

$$
\left(\int_{M} h^{\frac{2 \kappa}{\kappa-2}} d V\right)^{\frac{\kappa-2}{\kappa}} \leq C(\kappa) \operatorname{Vol}(M)^{\frac{\kappa-2}{\kappa}}\left(\int_{M}\left|\nabla^{M} h\right|^{2} d V+\operatorname{Vol}(M)^{-1} \int_{M} h^{2} d V\right)
$$

for any $\kappa>2$. From inequality (1) and (2), we have the following estimate:

Lemma 5. Suppose $u$ is a positive sub-solution of the equation $\Delta_{M} u \geq g u$ on a closed manifold $M$, where $g$ is a $L^{1}$ function satisfying the estimate $\|g\|_{L^{\frac{r}{2}}} \leq \bar{c} \operatorname{Vol}(M)^{\frac{2}{r}-\frac{2}{n}}$ for some $r>n$. Then $\|u\|_{0, M} \leq C_{p} \operatorname{Vol}(M)^{-\frac{1}{p}}\|u\|_{L^{p}}$ for $p>0$. The constant $C_{p}$ depends on $n, r, \bar{c}$ and $p$.

Proof. Multiply both sides of $\Delta_{M} u \geq g u$ by $u^{q-1}, q \geq 2$, and integrate over $M$. One gets

or

$$
\int_{M} u^{q-1} \Delta_{M} u d V \geq \int_{M} g u^{q} d V
$$

$$
-(q-1) \int_{M} u^{q-2}\left|\nabla^{M} u\right|^{2} d V \geq \int_{M} g u^{q} d V .
$$

Rewriting the left hand side and using Hölder inequality, it leads to

$$
\frac{4(q-1)}{q^{2}} \int_{M}\left|\nabla^{M} u^{\frac{q}{2}}\right|^{2} d V \leq\|g\|_{L^{\frac{r}{2}}}\left\|u^{q}\right\|_{L^{\frac{r}{r-2}}} .
$$

This together with the Sobolev inequality gives the following inequality in the case $n>2$ :

$$
\begin{aligned}
\left(\int_{M} u^{\frac{q}{2} \cdot \frac{2 n}{n-2}} d V\right)^{\frac{n-2}{n}} & \leq C(n)\left(\int_{M}\left|\nabla^{M} u^{\frac{q}{2}}\right|^{2} d V+\operatorname{Vol}(M)^{-\frac{2}{n}} \int_{M} u^{q} d V\right) \\
& \leq C(n)\left(c q\|g\|_{L^{\frac{r}{2}}}\left\|u^{q}\right\|_{L^{\frac{r}{r-2}}}+\operatorname{Vol}(M)^{\frac{2}{r}-\frac{2}{n}}\left\|u^{q}\right\|_{L^{\frac{r}{r-2}}}\right) \\
& \leq C q \operatorname{Vol}(M)^{\frac{2}{r}-\frac{2}{n}}\left\|u^{q}\right\|_{L^{\frac{r}{r-2}}}
\end{aligned}
$$


The constant $C$ depends on $n$ and $\bar{c}$. For $n=2$, we can choose $\kappa=\frac{r+2}{2}$ and similarly get

$$
\left(\int_{M} u^{\frac{q}{2} \cdot \frac{2 \kappa}{\kappa-2}} d V\right)^{\frac{\kappa-2}{\kappa}} \leq C q \operatorname{Vol}(M)^{\frac{2}{r}-\frac{2}{\kappa}}\left\|u^{q}\right\|_{L^{\frac{r}{r-2}}} .
$$

The constant $C$ depends on $\kappa$ and $\bar{c}$. Denote $\hat{n}=n$ for $n>2$ and $\hat{n}=\kappa$ for $n=2$. Then

$$
\left(\int_{M} u^{\frac{q \hat{n}}{\hat{n}-2}} d V\right)^{\frac{\hat{n}-2}{q \hat{n}}} \leq\left(C q \operatorname{Vol}(M)^{\frac{2}{r}-\frac{2}{\hat{n}}}\right)^{\frac{1}{q}}\|u\|_{L^{\frac{q r}{r-2}}} .
$$

If we denote $\left(\int_{M} u^{x} d V\right)^{\frac{1}{x}}$ by $\Psi(x)$, the inequality can be written as

$$
\Psi(q k) \leq\left(C q \operatorname{Vol}(M)^{\frac{2}{r}-\frac{2}{\tilde{n}}}\right)^{\frac{1}{q}} \Psi(q s),
$$

where $k=\frac{\hat{n}}{\hat{n}-2}$ and $s=\frac{r}{r-2}$. Because $r$ is greater than $\hat{n}$, the number $\gamma=\frac{k}{s}$ is greater than one and

$$
\Psi(\gamma x) \leq\left(C \frac{x}{s} \operatorname{Vol}(M)^{\frac{2}{r}-\frac{2}{\hat{n}}}\right)^{\frac{s}{x}} \Psi(x),
$$

for any $x \geq 2 s$. Choose $x=\gamma^{m-1} p$ for $p \geq 2 s$. One then has

$$
\begin{aligned}
\Psi\left(p \gamma^{m}\right) & \leq\left(C \frac{p \gamma^{m-1}}{s} \operatorname{Vol}(M)^{\frac{2}{r}-\frac{2}{\hat{n}}}\right)^{\frac{s}{p \gamma^{m-1}}} \Psi\left(p \gamma^{m-1}\right) \\
& \leq\left(\frac{C p}{s} \operatorname{Vol}(M)^{\frac{2}{r}-\frac{2}{\hat{n}}}\right)^{\frac{s}{p} \sum_{i=0}^{m-1} \frac{1}{\gamma^{2}}} \gamma^{\frac{s}{p} \sum_{i=0}^{m-1} \frac{i}{\gamma^{2}}} \Psi(p) .
\end{aligned}
$$

Let $m$ go to infinity and notice that

$$
\sum_{i=0}^{\infty} \frac{1}{\gamma^{i}}=\frac{k}{k-s} \quad \text { and } \quad-\frac{1}{k}+\frac{1}{s}=\frac{2}{\hat{n}}-\frac{2}{r} .
$$

It follows that

$$
\|u\|_{0, M} \leq C \operatorname{Vol}(M)^{-\frac{1}{p}}\|u\|_{L^{p}} \quad \text { for } p \geq 2 s
$$

The constant $C$ depends on $\hat{n}, r$ and $\bar{c}$. For general $p$, we first recall that

$$
\begin{aligned}
\left(\int_{M} u^{q k} d V\right)^{\frac{1}{k}} & \leq C q \operatorname{Vol}(M)^{\frac{2}{r}-\frac{2}{n}}\left(\int_{M} u^{q s} d V\right)^{\frac{1}{s}} \\
& =C q \operatorname{Vol}(M)^{\frac{1}{k}-\frac{1}{s}}\left(\int_{M} u^{q s} d V\right)^{\frac{1}{s}} .
\end{aligned}
$$


Therefore,

$$
\begin{aligned}
& \operatorname{Vol}(M)^{-\frac{1}{k q}}\left(\int_{M} u^{q k} d V\right)^{\frac{1}{k q}} \\
\leq & (C q)^{\frac{1}{q}}\left[\operatorname{Vol}(M)^{-\frac{1}{\lambda}}\left(\int_{M} u^{q s(1-\varepsilon) \lambda} d V\right)^{\frac{1}{\lambda}} \operatorname{Vol}(M)^{-\frac{1}{\mu}}\left(\int_{M} u^{q s \varepsilon \mu} d V\right)^{\frac{1}{\mu}}\right]^{\frac{1}{s q}}
\end{aligned}
$$

for $1>\varepsilon>0$ and $\frac{1}{\lambda}+\frac{1}{\mu}=1$. If we choose $\lambda$ satisfying $q s(1-\varepsilon) \lambda=q k$, it follows that

$$
\operatorname{Vol}(M)^{-\frac{\varepsilon}{k q}}\left(\int_{M} u^{k q} d V\right)^{\frac{\varepsilon}{k q}} \leq(C q)^{\frac{1}{q}} \operatorname{Vol}(M)^{-\frac{1}{\mu q s}}\left(\int_{M} u^{\mu q s \varepsilon} d V\right)^{\frac{1}{\mu q s}}
$$

That is,

$$
\operatorname{Vol}(M)^{-\frac{1}{k q}}\left(\int_{M} u^{k q} d V\right)^{\frac{1}{k q}} \leq(C q)^{\frac{1}{q \varepsilon}} \operatorname{Vol}(M)^{-\frac{1}{\mu q s \varepsilon}}\left(\int_{M} u^{\mu q s \varepsilon} d V\right)^{\frac{1}{\mu q s \varepsilon}}
$$

Let $q=2$ and $p=2 s \varepsilon \mu=\frac{2 k s \varepsilon}{k-s+s \varepsilon}$, then

$$
\operatorname{Vol}(M)^{-\frac{1}{2 k}}\left(\int_{M} u^{2 k} d V\right)^{\frac{1}{2 k}} \leq(2 C)^{\frac{1}{2 \varepsilon}} \operatorname{Vol}(M)^{-\frac{1}{p}}\left(\int_{M} u^{p} d V\right)^{\frac{1}{p}}
$$

By varying $\varepsilon$, we can choose $p$ to be any positive number. Combining inequalities (3) and (4) gives

$$
\|u\|_{0, M} \leq C_{p} \operatorname{Vol}(M)^{-\frac{1}{p}}\|u\|_{L^{p},} \quad \text { for } \quad p>0 .
$$

\section{Q.E.D.}

Remark: The constant on the right hand side of the inequality (1) or (2) is called the Sobolev constant on $M$. The quantity can be defined in a general Riemannian manifold. Since we only use (1) and (2) to derive the estimate, the lemma holds in general and the constant $C_{p}$ depends on the Sobolev constant on $M, r, \bar{c}$ and $p$.

From Lemma 5, We can get the following sup. norm estimate:

Theorem 5. Suppose that $u$ is a $W^{1,2}$ weak solution for $\Delta_{M} u=f$ on a closed Riemannian manifold $M$, where $f$ satisfies $\|f\|_{L^{\frac{r}{2}}}<\infty$, for some $r>n$. Then

$$
\|u\|_{0, M} \leq C\left(\operatorname{Vol}(M)^{-\frac{1}{2}}\|u\|_{L^{2}}+\operatorname{Vol}(M)^{\frac{2}{n}-\frac{2}{r}}\|f\|_{L^{\frac{r}{2}}},\right.
$$

where $C$ depends only on $r$ and the Sobolev constant on $M$. 
Proof. Define $\beta=\operatorname{Vol}(M)^{\frac{2}{n}-\frac{2}{r}}\|f\|_{L^{\frac{r}{2}}}$ and $\xi=\frac{1}{2}\left[\left(\frac{u}{\beta}\right)^{2}+1\right]$. It is easy to see that $\xi$ is a weak solution for

$$
\Delta_{M} \xi \geq \frac{f}{\beta} \frac{u}{\beta}=\frac{f}{\beta} \frac{u}{\beta} \frac{1}{\xi} \xi .
$$

Denote $g=\frac{f}{\beta} \frac{u}{\beta} \frac{1}{\xi}$. Because $\left|\frac{u}{\beta \xi}\right|<2$, one can prove

$$
\|g\|_{L^{\frac{r}{2}}} \leq \frac{2}{\beta}\|f\|_{L^{\frac{r}{2}}} \leq 2 \operatorname{Vol}(M)^{\frac{2}{r}-\frac{2}{n}} .
$$

It follows from Lemma 5 that

$$
\xi \leq C \operatorname{Vol}(M)^{-1}\|\xi\|_{L^{1}}
$$

Hence

$$
\left|\frac{u}{\beta}\right|^{2} \leq C \operatorname{Vol}(M)^{-1}\left[\frac{1}{2}\left\|\left(\frac{u}{\beta}\right)^{2}\right\|_{L^{1}}+\operatorname{Vol}(M)\right]
$$

Therefore,

$$
\begin{aligned}
|u| & \leq C \beta \sqrt{1+\operatorname{Vol}(M)^{-1}\left\|\left(\frac{u}{\beta}\right)^{2}\right\|_{L^{1}}} \\
& \leq C \sqrt{\beta^{2}+\operatorname{Vol}(M)^{-1}\left\|u^{2}\right\|_{L^{1}}} \\
& \leq C\left(\beta+\operatorname{Vol}(M)^{-\frac{1}{2}}\|u\|_{L^{2}}\right) \\
& =C\left(\operatorname{Vol}(M)^{\frac{2}{n}-\frac{2}{r}}\|f\|_{L^{\frac{r}{2}}}+\operatorname{Vol}(M)^{-\frac{1}{2}}\|u\|_{L^{2}}\right) .
\end{aligned}
$$

Again, the constant $C$ may change slightly in different places.

Q.E.D.

Suppose that $M_{\alpha}$ and $\rho$ are as defined in section 3 and section 4 . We need the following estimate in weighted norm to prove the main theorem.

Lemma 6. [3] For any given positive number $\nu$, there exists $\alpha_{0}$ such that the following estimate holds. Suppose that $\alpha<\alpha_{0}$ and $u$ is a $C^{2, \beta}$ function on $M_{\alpha}$, which satisfies $\int_{M_{\alpha}} u d V=0$. Then

$$
\|u\|_{0, M_{\alpha}} \leq C \varepsilon^{-\nu}\left\|\rho^{2} \Delta_{M_{\alpha}} u\right\|_{C_{\rho}^{0, \beta}\left(M_{\alpha}\right)},
$$

where the constant $C$ depends on $\nu$, but is independent of $\alpha$. 
Proof. Assume that the Lemma does not hold. Then there exists a sequence $\alpha_{j} \rightarrow 0$, its corresponding $\varepsilon_{j}, \rho_{j}$, and $u_{j} \in C^{2, \beta}\left(M_{\alpha_{j}}\right)$ which satisfies $\int_{M_{\alpha_{j}}} u_{j} d V=0$ and

$$
\left\|u_{j}\right\|_{0, M_{\alpha_{j}}} \geq j \varepsilon_{j}^{-\nu}\left\|\rho_{j}^{2} \Delta_{M_{\alpha_{j}}} u_{j}\right\|_{C_{\rho_{j}}^{0, \beta}\left(M_{\alpha_{j}}\right)} .
$$

We can normalize $u_{j}$ such that $\left\|u_{j}\right\|_{0, M_{\alpha_{j}}}=1$. It follows that

$$
\left\|\rho_{j}^{2} \Delta_{M_{\alpha_{j}}} u_{j}\right\|_{C_{\rho_{j}}^{0, \beta}\left(M_{\alpha_{j}}\right)} \leq \frac{1}{j} \varepsilon_{j}^{\nu} .
$$

On the other hand, by Theorem 5 we have

$$
\left\|u_{j}\right\|_{0, M_{\alpha_{j}}} \leq C\left[\operatorname{Vol}\left(M_{\alpha_{j}}\right)^{-\frac{1}{2}}\left\|u_{j}\right\|_{L^{2}}+\operatorname{Vol}\left(M_{\alpha_{j}}\right)^{\frac{2}{n}-\frac{2}{r}}\left\|\Delta_{M_{\alpha_{j}}} u_{j}\right\|_{L^{\frac{r}{2}}}\right] .
$$

Because $u_{j}$ satisfies $\int_{M_{\alpha_{j}}} u_{j} d V=0$, it implies

$$
\lambda_{1}\left(M_{\alpha_{j}}\right) \int_{M_{\alpha_{j}}} u_{j}^{2} d V \leq-\int_{M_{\alpha_{j}}}<\Delta_{M_{\alpha_{j}}} u_{j}, u_{j}>d V \leq\left\|\Delta_{M_{\alpha_{j}}} u_{j}\right\|_{L^{1}} .
$$

Remember that the Sobolev constant on $M_{\alpha_{j}}$ is bounded uniformly, the volume $\operatorname{Vol}\left(M_{\alpha_{j}}\right)$ is bounded uniformly from above and below, and $\lambda_{1}\left(M_{\alpha_{j}}\right)$ is bounded below by $\frac{1}{4} \lambda_{1}(L)$. Choose $r$ satisfying $-r+\frac{\nu r}{2} \geq-n$, and one obtains

$$
\begin{aligned}
1=\left\|u_{j}\right\|_{0, M_{\alpha_{j}}} & \leq C_{r}\left(\left\|\Delta_{M_{\alpha_{j}}} u_{j}\right\|_{L^{1}}+\left\|\Delta_{M_{\alpha_{j}}} u_{j}\right\|_{L^{\frac{r}{2}}}\right) \\
& \leq C_{r}\left[\int_{M_{\alpha_{j}}} \rho_{j}^{-r}\left(\rho_{j}^{2} \Delta_{M_{\alpha_{j}}} u_{j}\right)^{\frac{r}{2}} d V\right]^{\frac{2}{r}} \\
& \leq C_{r}\left[\int_{M_{\alpha_{j}}} \rho_{j}^{-r}\left(\frac{1}{j} \varepsilon_{j}^{\nu}\right)^{\frac{r}{2}} d V\right]^{\frac{2}{r}} \\
& \leq \frac{C_{r}}{j}\left(\int_{M_{\alpha_{j}}} \rho_{j}^{-r+\frac{\nu r}{2}} d V\right)^{\frac{2}{r}} \\
& \leq \frac{C_{r}}{j} .
\end{aligned}
$$

The constant $C_{r}$ may change in different places. Because the constant $C_{r}$ is independent of $j$, the above inequality leads to a contradiction. Therefore, the lemma must hold.

Q.E.D. 


\section{References.}

[1] R. Abraham \& J.E. Marsden \& T. Ratiu, Manifolds, tensor analysis, and applications, second ed., Springer-Verlag, New York, 1988.

[2] A. Butscher, Deformation theory on minimal Lagrangian submanifolds, Ph.D thesis, Stanford University, (2000).

[3] A. Butscher, Regularising a singular special Lagrangian variety, math.DG/0110053.

[4] A. Butscher, personal notes, (2000).

[5] M. Gross, Topological mirror symmetry, Invent. Math. 144 (2001), no. $1,75-137$.

[6] R. Harvey, Spinors and calibrations, Perspectives in Math. Vol. 9, Academic Press Inc., (1990).

[7] R. Harvey \& H.B. Lawson, Calibrated geometries, Acta Math. 148 (1982), 48-156.

[8] D. Joyce, On counting special Lagrangian homology 3-sphere, hepth/9907013, (1999).

[9] D. Joyce, Singularities of special Lagrangian fibrations and the SYZ conjecture, math.DG/0011179, 2000

[10] G. Lawlor, The angle criterion, Invent. Math. 95 (1989) 437-446.

[11] Y.I. Lee, Minimal and Lagrangian surfaces in Kähler- Einstein surfaces of negative scalar curvature, Comm. Anal. Geom. 2 (1994), no.4, 579592.

[12] Y.I. Lee, The deformation of Lagrangian minimal surfaces in KählerEinstein surfaces, J. Differential Geom. 50 (1998), no. 2, 299-330.

[13] R.C. McLean, Deformations of special Lagrangian submanifolds, Comm. Anal. Geom. 6 (1998), no. 4, 705-747.

[14] J. H. Michael \& L. Simon, Sobolev and mean-value inequalities on generalized submanifolds of $R^{n}$, Comm. Pure Appl. Math. 26 (1973), 361379 . 
[15] J.K. Moser, On the volume elements on manifolds, Trans. Amer. Math. Soc. 120 (1965), 280-296.

[16] D. Nance, Sufficient conditions for a pair of n-planes to be areaminimizing, Math. Ann. 279 (1987), 161-164.

[17] W.D. Ruan, Lagrangian torus fibration and mirror symmetry of CalabiYau hypersurface in toric variety, math.DG/0007028, 2000.

[18] R. Schoen, Lecture notes in geometric PDEs on manifolds, Course given in the spring of 1998 at Stanford University.

[19] R. Schoen \& J.G. Wolfson, Minimizing volume among Lagrangian submanifolds, in Differential Equations: La Pietra 1996, edited by Giaquinta, Shatah and Varadhan, Proc. of Symp. in Pure Math. 65 (1999), 181-199.

[20] R. Schoen \& J.G. Wolfson, Minimizing area among Lagrangian surfaces: the mapping problem, J. Differential Geom. 58 (2001), no. 1, 1-86.

[21] L. Simon, Lectures on geometric measure theory, Proc. Centre Math. Anal. Austral. Nat. Univ. Vol. 3, Canberra, (1983).

[22] L. Simon, Lecture notes in PDE theory, Course given in 1997 at Stanford University.

[23] A. Strominger \& S.T. Yau \& E. Zaslow, Mirror symmetry is T-duality, Nuclear Phys. B 479 (1996) 243-259.

[24] S.T. Yau, On the Ricci curvature of a compact Kähler manifold and the complex Monge-Ampère equations, Comm. pure appl. math. 31 (1978), 339-411.

NATIONAL TAIWAN UNIVERSITY

Department of Mathematics

TAIPEI, TAIWAN

R.O.C.

yilee@math.ntu.edu.tw

Received November 15, 2001. 